\title{
Advances in Clinical Cardiology 2016: A Summary of the Key Clinical Trials
}

\author{
Alastair Gray · Conor McQuillan · Ian B. A. Menown
}

Received: April 10, 2017 / Published online: May 23, 2017

(C) The Author(s) 2017. This article is an open access publication

\section{ABSTRACT}

Introduction: The findings of many new cardiology clinical trials over the last year have been published or presented at major international meetings. This paper aims to describe and place in context a summary of the key clinical trials in cardiology presented between January and December 2016.

Methods: The authors reviewed clinical trials presented at major cardiology conferences during 2016 including the American College of Cardiology (ACC), European Association for Percutaneous Cardiovascular Interventions (EuroPCR), European Society of Cardiology (ESC), European Association for the Study of Diabetes (EASD), Transcatheter Cardiovascular Therapeutics (TCT), and the American Heart Association (AHA). Selection criteria were trials with a broad relevance to the cardiology community and those with potential to change current practice.

Results: A total of 57 key cardiology clinical trials were identified for inclusion. Here we describe and place in clinical context the key

Enhanced content To view enhanced content for this article go to http://www.medengine.com/Redeem/ 0B18F0603C0130FD.

A. Gray · C. McQuillan · I. B. A. Menown ( ()

Craigavon Cardiac Centre, Southern Trust,

Craigavon, Northern Ireland, UK

e-mail: ian.menown@southerntrust.hscni.net findings of new data relating to interventional and structural cardiology including delayed stenting following primary angioplasty, contrast-induced nephropathy, management of jailed wires, optimal duration of dual antiplatelet therapy (DAPT), stenting vs bypass for left main disease, new generation stents (BioFreedom, Orsiro, Absorb), transcatheter aortic valve implantation (Edwards Sapien XT, transcatheter embolic protection), and closure devices (Watchman, Amplatzer). New preventative cardiology data include trials of bariatric surgery, empagliflozin, liraglutide, semaglutide, PCSK9 inhibitors (evolocumab and alirocumab), and inclisiran. Antiplatelet therapy trials include platelet function monitoring and ticagrelor vs clopidogrel for peripheral vascular disease. New data are also presented in fields of heart failure (sacubitril/valsartan, aliskiren, spironolactone), atrial fibrillation (rivaroxaban in patients undergoing coronary intervention, edoxaban in DC cardioversion), cardiac devices (implantable cardioverter defibrillator in non-ischemic cardiomyopathy), and electrophysiology (cryoballoon vs radiofrequency ablation).

Conclusion: This paper presents a summary of key clinical cardiology trials during the past year and should be of practical value to both clinicians and cardiology researchers.

Keywords: Ablation;

Anticoagulant; Antiplatelet; Acute coronary syndrome; Atrial 
fibrillation; Bioabsorbable polymer; Drug eluting balloon; Lipids; Prevention; Revascularization

\section{INTRODUCTION}

Over the past year, many trials with the potential to change practice and impact on clinical guidelines have been presented at international meetings including the American College of Cardiology (ACC), European Association for Percutaneous Cardiovascular Interventions (EuroPCR), European Society of Cardiology (ESC), European Association for the Study of Diabetes (EASD), Transcatheter Cardiovascular Therapeutics (TCT), and the American Heart Association (AHA). In this paper the authors describe new clinical data placed in the context of acute coronary syndrome (ACS), interventional cardiology, heart failure, atrial fibrillation, electrophysiology, and coronary prevention.

\section{METHODS}

The results of major clinical trials in cardiology presented at major conferences in 2016 were reviewed by the authors in addition to a search of PubMed, Medline, Cochrane library, and Embase. Search terms included "acute coronary syndrome," "atrial fibrillation," "coronary prevention," "electrophysiology," "heart failure," and "interventional cardiology." Trials were selected on the basis of relevance to the cardiology practice and the potential to guide further phase 3 research. This article is based on previously conducted studies and does not involve any new studies of human or animal subjects performed by any of the authors.

\section{ADVANCES IN INTERVENTIONAL CARDIOLOGY}

Coronary revascularization via primary percutaneous coronary intervention (PCI) is the gold standard treatment for patients with ST elevation myocardial infarction (STEMI) but may be complicated by slow or no-reflow. Registry data have suggested that delayed stent implantation, after restoration of antegrade flow, may reduce distal embolization thereby potentially improving clinical outcomes. The DANAMI 3DEFER (Deferred versus conventional stent implantation in patients with STEMI) trial randomized 1215 patients with STEMI to standard PCI (612) or deferred stent implantation (603) after $48 \mathrm{~h}$ [1]. There was no difference in primary endpoint (composite of all-cause mortality, hospital admission for heart failure, recurrent infarction, and unplanned target vessel revascularization) with deferred vs conventional stent implantation $(17 \%$ vs $18 \%$; $p=0.92$ ). Thus while stenting at the time of primary PCI remains appropriate for the majority of patients (avoiding the need for a second procedure), deferred PCI in certain settings such as high thrombus burden appears reasonable.

Contrast-induced nephropathy (CIN) remains a frequent complication of coronary angiography [2]. The CARIN (Safety and Efficacy of CMX-2043 for Periprocedural Injury Protection in Subjects Undergoing Coronary Angiography at Risk of Radio-contrast Induced Nephropathy) trial assigned 3503 patients undergoing coronary angiography to three incremental doses of CMX-2043 (an $\alpha$-lipoic acid analogue aimed at reducing ischemia-reperfusion injury) vs placebo [3]. However, no significant reduction in the incidence of the primary endpoint (acute kidney injury at 4 days) was observed with CMX-2043. Thus preventative strategies continue to focus on optimization of preprocedure hydration and withholding of nephrotoxic medications [2].

For decades, PCI fellows have been taught that hydrophilic guidewires should not be "jailed" in side branches behind main vessel stent struts because of concerns that their polymer coating might be stripped off on wire removal. At EuroPCR 2016, this dogma was debunked by a study of 235 patients undergoing bifurcation PCI who were randomized to either a polymer-coated (PC) or non-polymer-coated (non-PC) guidewire for side-branch access and jailing [4]. Initial side branch wiring was significantly faster with PC wires (19 vs $42 \mathrm{~s}$; 
$p=0.05$ ). In subsequent wire evaluation by stereomicroscopy, PC wires had markedly less damage $(1.7 \%$ PC wires damaged [all only mildly damaged vs $55 \%$ non-PC wires damaged, $41 \%$ of which were moderately to severely damaged]; $p=0.001)$. Contributing factors to wire damage included main vessel lesion length and jailed wire length. This small but important trial has been tipped to change worldwide PCI practice.

Radial access popularity continues to grow worldwide given its lower bleeding risk. However, interim data at EuroPCR from a study of 191 patients undergoing racial access PCI reported upper extremity dysfunction in the intervention arm (as defined by a combination of Doppler, volumetry, and sensory testing) in $74.9 \%$ of cases. Those with upper extremity dysfunction were more likely to have radial artery occlusion $(9.8 \%$ vs $0 \% ; p<0.001)$ and to be younger (63.4 vs 67.5 years; $p=0.01$ ) [5]. Final data from 500 patients are expected in mid-2017.

The pathophysiology of saphenous vein graft (SVG) atherosclerosis appears different to native coronary artery disease. BASKET-SAVAGE (Drug-eluting vs bare metal stents in saphenous vein grafts) assessed the efficacy and safety of drug-eluting stents (DES) vs bare metal stents (BMS) in SVG PCI [6]. Despite the trial stopping early because of slow enrollment, those randomized to DES $(n=89)$ vs BMS $(n=84)$ had markedly lower primary endpoint of major adverse cardiac events (MACE) at 12 months $(2.3 \%$ vs $17.9 \% ; p<0.001)$ which was maintained at 3 years $(12.4 \%$ vs $28.9 \% ; p=0.0012)$. These differences were largely driven by target lesion revascularization (TLR) and non-fatal MI, with no significant difference in mortality.

It is recognized that the improved clinical outcomes with newer compared with older generation stents may be related to improved stent design as well as polymer/drug factors [7]. It is thus of interest that the NORSTENT (Norwegian Coronary Stent Trial) randomized 9013 patients (1:1) undergoing PCI to DES $(82.9 \%$ everolimus, $13.1 \%$ zotarolimus) or contemporary BMS [8]. Of note, two-stent bifurcation PCI was excluded. There was no difference in the primary outcome of death or MI at 6 years
$(16.6 \%$ vs $17.1 \% ; p=0.66)$ in keeping with BASKET-PROVE results [9]. However, DES was associated with reduced TLR $(5.3 \%$ vs $10.3 \%$; $p<0.001)$ and, interestingly, a reduced incidence of definite stent thrombosis $(0.8 \%$ vs $1.2 \% ; p=0.0498)$. Thus contemporary DES improve overall PCI outcomes although contemporary BMS remain a reasonable option in certain clinical settings.

Given the potential benefits of 30 months' dual antiplatelet therapy (DAPT) seen in the DAPT trial [10], optimal duration of DAPT was a prominent discussion point at the European Society Congress (ESC). The NIPPON (Nobori Dual Antiplatelet Therapy as Appropriate Duration) trial randomized patients undergoing PCI with the Nobori ${ }^{\circledR}$ bioabsorbable abluminal-coated stent to short (6 months) vs long (18 months) duration of DAPT (primarily clopidogrel) [11]. Interim analysis from 2772 of 3775 patients showed no significant difference in incidence of NACCE (net adverse clinical and cerebral events) $(1.92 \%[n=1355]$ vs $1.45 \%$ $[n=1371]$ for 6 and 18 months, respectively), bleeding, or stent thrombosis, although results must be interpreted with caution until full trial data are available especially given the open-label design and lower than expected events rate which is likely to have reduced statistical power.

Provisional main vessel PCI is the recommended technique for most bifurcation lesions [12]. However, when a two-stent strategy is required, opinion is divided on the optimal technique. In the BBK-II (Bifurcations Bad Krozingen II) angiographic trial, 300 patients undergoing bifurcation stenting with DES were randomized (1:1) to culotte vs TAP (T-and-Protrude) stenting [13]. Culotte was superior with respect to the primary endpoint of 9-month percentage diameter stenosis $(21 \%$ vs $27 \%$; $p=0.038)$ and binary restenosis $(6.5 \%$ vs $17 \%$; $p=0.006)$. In keeping with previous data [14] (which showed culotte to be superior to crush), the BBK-II trial suggests that if a two-stent strategy is required, culotte should be the preferred technique in most patients.

While $N$-acetylcysteine (NAC) was ultimately disappointing in trials of prevention of contrast nephropathy, its potential antioxidant properties were evaluated in the new setting of infarct 
size reduction during primary PCI for STEMI. The NACIAM trial ( $N$-AcetylCysteine in Acute Myocardial infarction) randomized 112 patients to $48 \mathrm{~h}$ of intravenous (IV) NAC administration vs placebo. All patients also received glyceryl trinitrate intravenously. Use of NAC was associated with reduction in infarct size by cardiac magnetic resonance (CMR) (mean infarct size $5 \%$ vs $10 \%$ of myocardium; $p=0.02$ ), reduced transmural infarct ( $54 \%$ vs $79 \% ; p=0.02)$, and improved myocardial salvage $(60 \%$ vs $27 \%$; $p=0.001$ ) [15]. Given these interesting results, a larger trial is planned.

Invasive diagnostic coronary angiography, when undertaken according to conventional referral pathways in patients with suspected angina, typically only demonstrates significant coronary disease in around one-third of cases. New 2016 trial data suggests that greater use of CT coronary angiography (CTCA) or CMR may enable improved triage and reduce unnecessary invasive angiography $[16,17]$.

Routine CTCA (Fig. 1) has previously been found to be superior to standard non-invasive protocols for chest pain assessment and triage [18]. The CONSERVE (COronary Computed Tomographic ANgiography for SElective Cardiac Catheterization Relation to CardioVascular Outcomes and Economics) trial randomized 1503 stable patients who had suspected coronary artery disease and guideline indications for

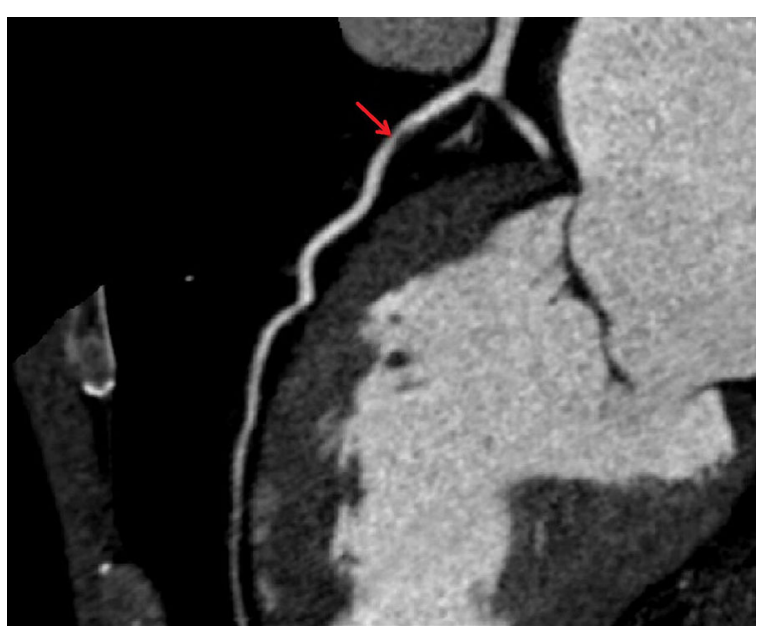

Fig. 1 Computed tomography coronary angiography showing a left anterior descending (LAD) artery stenosis angiography either directly to invasive coronary angiography (ICA) or to initial CTCA (only followed by ICA if the CTCA was positive). The conservative approach (initial CTCA) was associated with a $78 \%$ reduction in referral for ICA and a $50 \%$ reduction in cost. Despite the lower use of ICA in the conservative group, there was no significant difference in MACE (death, MI, unstable angina, stroke, urgent revascularization, or CV hospitalization) between the two approaches (each 4.6\%) [16].

CMR has been shown to have superior sensitivity and negative predictive valve compared with myocardial perfusion scintigraphy (MPS) for detection of angiographically significant coronary disease [19]. In the CE-MARC 2 (Clinical Evaluation of Magnetic Resonance Imaging in Coronary Heart Disease 2) trial, 1202 stable patients with clinically suspected angina and pretest probability score of $10-90 \%$ were randomized (1:2:2) to conventional guidelines (based on National Institute for Health and Care Excellence), CMR, or MPS to guide need for invasive angiography. By 12 months, angiography was undertaken in $43 \%, 18 \%$, and $16 \%$ of conventional, CMR and MPS groups, respectively. Significant angiographic findings (requiring intervention) were observed in $12.1 \%$, $9.8 \%$, and $8.7 \%$ of the three groups, respectively, although there was no significant difference in MACE events between groups [17]. Unnecessary angiography (absence of significant stenosis measured by fractional flow reserve or quantitative coronary angiography) occurred in $28.8 \%, 7.5 \%$, and $7.1 \%$ of the three groups, respectively.

\section{Coronary Artery Bypass vs PCI Revascularization}

Treatment of unprotected left main coronary artery disease by PCI has increased significantly on the basis of findings from small studies, subgroup analyses, and observational data, but adequately powered studies were awaited. At TCT 2016, findings of two key trials were presented.

EXCEL (Evaluation of Xience versus Coronary Artery Bypass Surgery for Effectiveness of 
Left Main Revascularization) randomized 1905 patients with significant left main disease (Syntax 32 or lower) to PCI with Xience everolimus-eluting stents or to CABG (voronary artery bypass grafting) [20]. The incidence of the primary composite endpoint of death, MI, and stroke at a median of 3 years was similar with PCI and CABG (15.4\% vs $14.7 \% ; p=0.02$ for non-inferiority). PCI was associated with lower death, MI, or stroke at the earlier time point of 30 days ( $4.9 \%$ vs $7.9 \% ; p=0.008$ for superiority) and a lower incidence of bleeding, arrhythmia, renal failure, and infection. Ischemia-driven revascularization was more common with PCI at 3 years $(12.6 \%$ vs $7.5 \% ; p<0.001)$ but thrombosis (definite stent thrombosis or graft occlusion) was less common with PCI $(0.7 \%$ vs 5.4\%; $p<0.001)$.

The NOBLE (Nordic-Baltic-British left main revascularisation) study randomized (1:1) 1201 patients with significant left main disease and no more than three non-left main, non-complex lesions to PCI $(n=598)$ or CABG $(n=603)$. The incidence of the primary endpoint of death, MI (excluding procedural MI), stroke, and revascularization up to 5 years (median 3 years) was higher in PCI than CABG [121 (29\%) vs $81(19 \%)$ patients; $p=0.0066$ ] but there was no significant difference in mortality [21]. Need for revascularization was more common with PCI than CABG ( $16 \%$ vs $10 \% ; p=0.032)$ but this was driven by de novo, non-left main lesions. Unlike the Syntax trial left main subset [22], in NOBLE, the baseline Syntax score did not predict outcome for those undergoing PCI and an unexpectedly high event rate was seen in those undergoing PCI in the low (1-22) Syntax subgroup. Further detailed analysis is planned to evaluate if this finding was due to specific anatomic subsets, specific PCI strategies, or other factors [23].

Although EXCEL and NOBLE outcomes appear disparate, this is likely due to differences in trial design. EXCEL included large procedural MI (creatine kinase MB greater than ten times upper normal) within the definition of MI, whereas NOBLE excluded procedural MI. EXCEL had a shorter follow-up than NOBLE which may be relevant since differences favoring CABG emerge between 3 and 5 years. EXCEL implanted only second-generation DES, whereas NOBLE included $11 \%$ of patients with first-generation DES before the new generation Biomatrix stent was mandated. After adjusting for these differences, the trial outcomes appear similar and in keeping with prior CABG vs PCI trial data.

A criticism of some studies is that patients undergoing study CABG have higher than usual rates of bilateral internal thoracic [mammary] artery (ITA) grafting which might be associated with better patency. The Arterial Revascularization Trial (ART) enrolled 3102 patients and randomly assigned $(1: 1)$ to single $(n=1554)$ or bilateral $(n=1548)$ ITA grafting. At 5 years, the rate of death $(8.7 \%$ vs $8.4 \% ; p=0.77)$ and composite of death from any cause, MI, or stroke $(12.2 \%$ vs $12.7 \% ; p=0.69)$ did not differ, but rates of sternal wound complications were higher with bilateral ITA grafts $(3.5 \%$ vs $1.9 \%$; $p=0.005$ ) [24]. However, longer follow-up is needed to evaluate the effect of presumed long-term patency of ITA vs vein grafting on clinical outcomes and 10-year follow-up is planned.

\section{New Generation Stents}

A short duration of DAPT post PCI is desirable in many patients including those at high bleeding risk. The BioFreedom stent facilitates a short 1-month course of DAPT since, instead of having a polymer layer, it has a novel microporous abluminal surface (Fig. 2) which enables controlled release of Biolimus A9 achieving therapeutic local tissue concentrations for 28 days post implantation [25]. The LEADERS-FREE trial randomized 2466 high-bleeding-risk patients to receive the BioFreedom drug-coated stent (DCS) or a bare metal stent (BMS) followed by 1-month DAPT. At 2-year follow-up BioFreedom was associated with a $46 \%$ reduction in the primary efficacy endpoint of clinically driven TLR $(6.8 \%$ vs $12.0 \%$ patients, respectively; hazard ratio $0.54 ; 95 \%$ CI $0.41-0.72 ; p<0.0001$ ) and a $20 \%$ reduction in the primary safety endpoint of cardiac death, stent thrombosis, or MI (12.6\% DCS vs $15.3 \%$ BMS patients; HR 0.80; 95\% CI 0.64-0.99; 


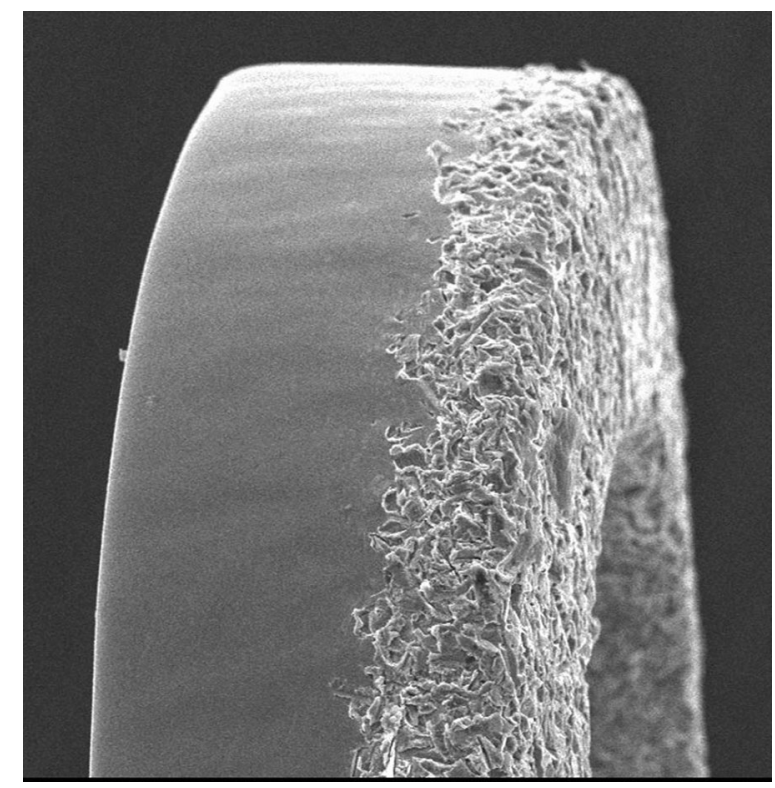

Fig. 2 BioFreedom stainless steel stent which incorporates a novel microporous abluminal surface. Reproduced with permission from Biosensors

$p=0.039$ ) (Fig. 3) [26]. Current use of BMS is now largely confined to patients thought likely to require short courses of DAPT and LEADERS FREE suggests that drug-coated stents will likely replace BMS for this indication also. The LEADERS FREE II registry is ongoing evaluating outcomes of BioFreedom in a combined US and global cohort.

For stents requiring a polymer for drug elution, use of a biodegradable polymer may reduce risk of very late stent thrombosis vs a permanent polymer [27]. The ultra-thin strut $(60 \mu \mathrm{m})$ cobalt-chromium Orsiro stent (Biotronik, Berlin, Germany) which has a passive silicon carbide layer overlaid by a biodegradable PLLA polymer releasing sirolimus was previously found to have similar overall clinical outcomes vs Xience (Abbott, Illinois, USA) and superior outcomes in the STEMI subgroup [28]. PCI in chronic total occlusion (CTO) is another challenging subgroup, typically associated with longer stent length and higher TLR. PRISON IV (Bioresorbable polymer-based sirolimus-eluting stent versus a durable polymer-based everolimus-eluting stent in patients with coronary artery chronic total occlusions) randomized 330 patients with successfully crossed chronic total occlusions to Orsiro vs Xience. Surprisingly, Orsiro was associated with greater in-segment late lumen loss $(0.13$ vs $0.02 \mathrm{~mm}$; primary non-inferiority endpoint not met) and more frequent binary restenosis $(8.0 \%$ vs $2.1 \% ; p=0.028$ ) at 9 months [29]. However, PRISON IV was underpowered to evaluate incidence of very late stent thrombosis and further studies are warranted.

The Absorb stent, a bioresorbable vascular scaffold (BVS), fully resorbs in 3 years. It was hoped the absence of a permanent stent would be associated with improved coronary vasomotion (reported to be impaired after first-generation DES) [30] and reduced risk of very late stent thrombosis. Significant expectation thus awaited findings of ABSORB II (Comparison of an everolimus-eluting bioresorbable scaffold with an everolimus-eluting metallic stent) in which 501 patients with one to two non-complex lesions undergoing PCI were randomized (2:1) to BVS vs Xience. At 3 years, disappointingly there was no significant difference in the co-primary endpoint of vasomotor reactivity after nitroglycerin, BVS had greater late lumen loss $\left(0.37\right.$ vs $0.25 \mathrm{~mm}$; $\left.p_{\text {non-inferiority }}=0.78\right)$, and of concern in the BVS group were eight definite scaffold thromboses (one acute, one subacute, six very late) and one probable late scaffold thrombosis vs zero with Xience $(p=0.0331)$ [31]. ABSORB II raised concerns that long duration (2-3 years) DAPT may be necessary after BVS and suggested that further design iteration and clinical study are required before BVS is considered suitable for widespread use.

\section{Intracoronary Diagnostics}

Diagnostic PCI is usually angiographically guided alone, although it is recognized that intravascular ultrasound (IVUS) guidance may be associated with decreased MACE. While optical coherence tomography (OCT) produces superior resolution (Fig. 4), equivocal or superior data guiding PCI is lacking. ILUMIEN III (OPTIMIZE PCI) sought to clarify this by randomizing (1:1:1) 450 patients to OCT, IVUS, or 


\section{A Primary Safety Endpoint}

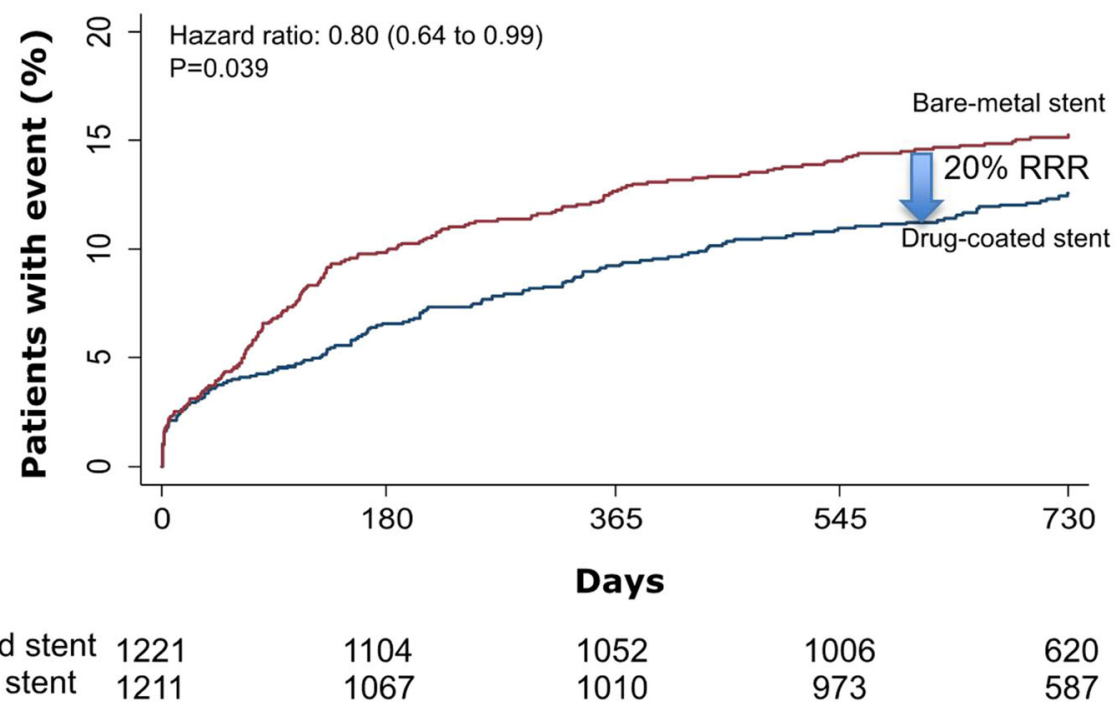

No. at risk

Drug-coated stent 122

Bare-metal stent 1211
$1067 \quad 1010$

587

\section{B Primary Efficacy Endpoint}

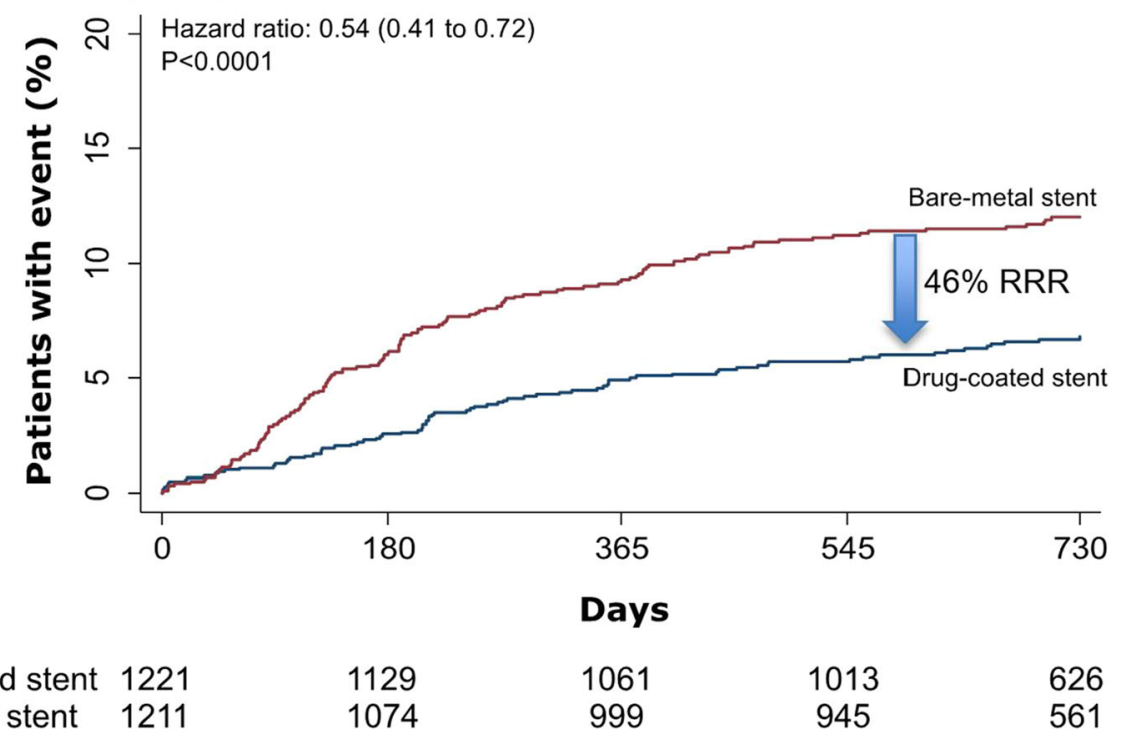

Fig. 3 Two-year follow-up of the LEADERS-FREE trial showing the BioFreedom drug-coated stent vs bare metal stenting to be associated with significant reduction in a the primary safety endpoint of cardiovascular death, stent

angiography with final OCT acquisitions for the primary endpoint of post-PCI minimal stent area (MSA). OCT guidance was non-inferior to IVUS guidance but not superior to IVUS or angiography alone [32]. Efficacy of OCTguided PCI to improve event-free survival after thrombosis, or myocardial infarction and $\mathbf{b}$ the primary efficacy endpoint of clinically driven target lesion revascularization. $R R R$ relative risk reduction. Reproduced with permission from Dr. P Urban

DES will be evaluated in the upcoming ILUMIEN IV trial.

Fractional flow reserve (FFR) is a guideline-recommended technique for guiding the use of PCI based on findings from multiple trials such as DEFER, FAME, and FAME II [33-35]. It 


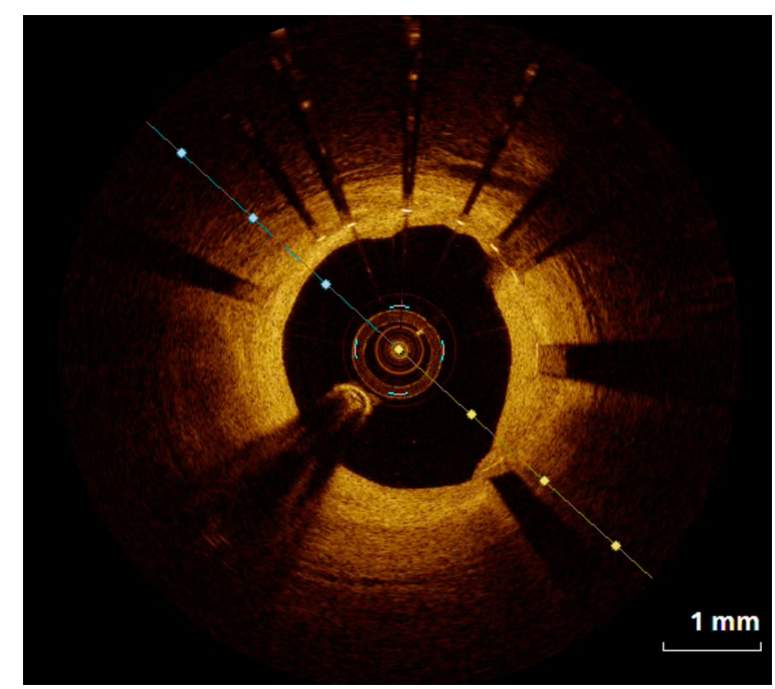

Fig. 4 Coronary artery optical coherence tomography

was thus surprising that the FUTURE trial [36] (Does FFR-guided treatment strategy in patients with multivessel CAD improve prognosis compared to traditional treatment), which planned to enroll 1728 patients, was halted prematurely after interim analysis suggested a higher 12 -month mortality in the FFR group (4\% vs $2 \% ; p=0.02$ ). Subsequent follow-up of 797 reaching 12-month follow-up showed a small numerical excess in all-cause mortality, but this was no longer significant $(p=0.07)$ and thought likely to be a spurious finding.

\section{Calcified Lesion Preparation}

With ever-older patients coming for PCI, strategies to adequately prepare heavily calcified lesions are increasingly needed. The novel technique of lithoplasty (high energy ultrasound waves) was evaluated in 60 patients undergoing PCI in the DISRUPT CAD (Shockwave Coronary Rx Lithoplasty $^{\circledR}$ Study trial). No angiographic complications were reported and OCT confirmed circumferential calcium fracture and luminal gain [37]. Larger studies with longer follow-up are required to assess the impact on clinical outcomes.

\section{Advances in Structural Cardiology}

Transcatheter aortic valve implantation (TAVI) is now an accepted treatment method for patients with severe aortic stenosis who are at high risk for surgical aortic valve replacement (SAVR) based on data from the Placement of Aortic Transcatheter Valves (PARTNER) study [38]. The PARTNER 2 trial aimed to evaluate TAVI in the setting of severe aortic stenosis and intermediate risk. In total, 2032 patients, of whom $81.3 \%$ had an STS [Society of Thoracic Surgeons] score between 4-8, were randomized (1:1 ratio) to TAVI (Edwards Lifesciences Sapien XT) or SAVR [39]. TAVI was non-inferior to SAVR with regard to the primary endpoint of death or disabling stroke at 2 years $(19.3 \%$ vs $21.1 \% ; p=0.001)$ and the transfemoral access TAVI cohort had lower rate of death or disabling stroke than SAVR (hazard ratio 0.79; 95\% CI 0.62-1.00; $p=0.05$ ).

While PARTNER 2 supports consideration of TAVI as an alternative to SAVR for severe aortic stenosis and intermediate risk patients, careful patient selection is still required as highlighted by data from the German Aortic Valve Registry [40] where, among 5997 intermediate risk patients (logistic EuroSCORE I of 10-20\%) undergoing isolated valve treatment, higher mortality rates were reported for TAVI vs SAVR, in hospital (3.8\% vs $2.6 \% ; p=0.02)$, at 30 days $(4.6 \%$ vs $3.2 \% ; p=0.01)$ and at 1 year $16.6 \%$ vs $8.9 \% ; p=0.001)$. Confounding factors including frailty and other co-morbidities may not have been fully accounted for, but intermediate to lower risk patient groups require further study.

As TAVI implant numbers increase, growing concern surrounds the incidence of transcatheter heart valve thrombosis. At ESC 2016, data were presented from a cohort of 405 intermediate risk patients (mean STS score 5.3) undergoing TAVI with Edwards Sapien XT or Sapien 3 who were subsequently evaluated by cardiac CT 1-3 months later for evidence of hypoattenuated leaflet thickening suggesting valve thrombosis [41]. Overall, valve thrombosis was observed in 28 (7\%) patients, of whom five had clinically obstructive symptoms but 23 were subclinical. Valve thrombosis was more common in those not receiving warfarin $(10.7 \%$ vs $1.8 \%$; risk ratio 6.09 ; $95 \%$ CI $1.86-19.84$ ) and with larger valves. Treatment with warfarin effectively resolved valve thrombosis and 
normalized function in $85 \%$ of patients. Data regarding optimal antithrombotic therapy post TAVI is limited, but ongoing randomized trials such as the Global Study Comparing a Rivaroxaban-based Antithrombotic Strategy to an Antiplatelet Strategy After Transcatheter Aortic Valve Replacement to Optimize Clinical Outcomes (GALILEO: NCT02556203) study may guide preferred antithrombotic strategy.

Embolism of atherothrombotic debris is common following TAVI. The SENTINEL trial tested whether transcatheter embolic protection (TCEP) during TAVI would decrease periprocedural neurological events and cerebral infarction. In SENTINEL, 363 high risk patients undergoing TAVI were randomized $(2: 1)$ to receive TCEP or routine (non-TCEP) care. Evidence captured embolic debris in 99\% of patients receiving TCEP. There was a small, non-significant increase in vascular complications with TCEP placement (8.6\% vs $5.9 \%)$. At 30 days, numerically lower MACCE $(7.3 \%$ vs $9.9 \%)$ and numerically lower strokes $(5.6 \%$ vs 9.1\%) were reported with TCEP, although these did not reach statistical significance [42]. In a subgroup undergoing brain MRI within 1 week, new lesion volume was numerically smaller with TCEP (102.8 vs $178.0 \mathrm{~mm}^{3} ; p=0.33$ ) but this did not reach statistical significance. Results are thus encouraging but further studies are needed. The degree of confirmed embolism detected also highlights the importance of avoiding overly aggressive device manipulation within the aortic valvar complex.

\section{Closure Devices}

Extended follow-up from PROTECT AF (Watchman Left Atrial Appendage System for Embolic Protection in Patients With AF) and PREVAIL (Prospective randomized evaluation of the Watchman Left Atrial Appendage Closure device in patients with atrial fibrillation vs long-term warfarin therapy) were presented at TCT 2016 as part of the CAP (Continued Access to PROTECT AF) and CAP2 (Continued Access to PREVAIL) registries [43] (Fig. 5). A total of 732 device patients were followed up for 5 and 3 years, respectively. Baseline predicted stroke

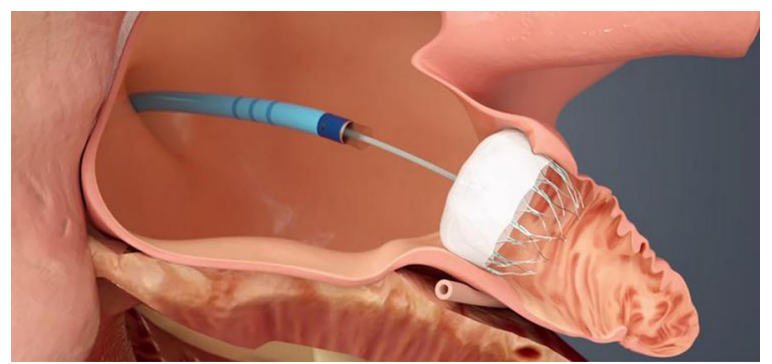

Fig. 5 Watchman left atrial appendage closure device Reproduced with permission from Boston Scientific

risk was $3.6 \%$ based on a median CHA2DS2VASc score of 3.4. Procedural complications included major bleeding (2.3\%), stroke $(1.0 \%)$, and device embolization (0.5\%). Risk ratios for ischemic stroke, hemorrhagic stroke, and major bleeding post-procedure were $1.35,0.16$, and 0.62 , respectively. The extended CAP follow-up suggested that Watchman is associated with a similar stroke risk reduction to that which would be expected with oral anticoagulants.

The significance and treatment of patent foramen ovale (PFO) in patients with cryptogenic stroke has been controversial. RESPECT (Closure of Patent Foramen Ovale vs Medical Therapy after Cryptogenic Stroke) 10-year outcome data followed 980 patients with cryptogenic stroke, randomized 1:1 to AMPLATZER PFO Occluder device vs medical management between 2003 and 2011 [44]. PFO closure using the AMPLATZER device was associated with a $45 \%$ risk reduction $(p=0.046)$ in recurrent ischemic stroke. There were no incidents of device embolization or thrombosis, and major vascular complications were low (0.9\%). PFO closure with the AMPLATZER device in appropriate patients thus appears to be associated with superior long-term outcomes compared with medical management alone.

\section{ADVANCES IN CARDIOVASCULAR PREVENTION}

\section{Diabetes Strategies}

The STAMPEDE (Surgical Therapy And Medications Potentially Eradicate Diabetes Efficiently) 
trial (NCT00432809) has previously reported short-term improvement in outcomes for overweight patients with poorly controlled diabetes randomized to bariatric surgery plus medical therapy $(n=100)$ or to medical therapy alone $(n=50)$, but long-term results were awaited with interest [45].

At 5 years, bariatric surgery was associated with greater weight reduction in body weight $(-23 \%,-19 \%$, and $-5 \%$ in the gastric bypass, sleeve gastrectomy, and medical therapy groups, respectively), triglyceride level $(-40 \%$, $-29 \%$, and $-8 \%$ ), and a greater achievement of the primary endpoint of normoglycemia defined as HbA1C $\leq 6.0 \%$ (29\%, 23\%, and 5\%). While such metabolic improvements would hopefully be associated with improved CV outcomes, STAMPEDE was not powered to assess this and larger studies of this promising technique are planned.

While older hypoglycemic treatments for diabetes have failed to show clear $\mathrm{CV}$ benefit, The sodium-glucose cotransporter 2 inhibitor empagliflozin did show reduction in CV death, but the mechanism was unclear given the absence of reduction in MI or stroke [25].

While it was hoped that GLP-1 receptor agonists would be beneficial, the previous Evaluation of Lixisenatide in Acute Coronary Syndrome (ELIXA) trial in patients with diabetes and a recent ACS failed to show CV benefit [46]. The Liraglutide Effect and Action in Diabetes: Evaluation of Cardiovascular Outcome Results (LEADER) trial randomized 9340 patients with diabetes (HbA1c $\geq 7 \%)$ and high $\mathrm{CV}$ risk to liraglutide $1.8 \mathrm{mg}$ once daily or placebo [47]. Liraglutide was associated with a significant reduction in the primary outcome of CV death, MI, or stroke (13.0\% vs $14.9 \%$, HR $0.87,95 \%$ CI $0.78-0.97 ; p=0.01)$, all-cause mortality $(8.2 \%$ vs $9.6 \% ; p=0.02)$, CV death $(4.7 \%$ vs $6.0 \% ; p=0.007)$, and $\mathrm{MI}(6.3 \%$ vs $7.3 \% ; p=0.046)$. No increase in heart failure was reported (unlike with some DPP-4 inhibitors). Subsequently, semaglutide, which has an extended half-life allowing once-weekly administration, was evaluated in the Trial to Evaluate Cardiovascular and Other Long-term Outcomes with Semaglutide in Subjects with Type 2 Diabetes (SUSTAIN-6) which randomized 2735 patients $(1: 1: 1: 1)$ to either $0.5 \mathrm{mg}$ or $1.0 \mathrm{mg}$ of once-weekly subcutaneous administration of semaglutide or volume-matched placebo. Semaglutide was associated with a significant reduction in the primary outcome of CV death, MI, or stroke [6.6\% vs 8.9\%; hazard ratio 0.74 ; $95 \%$ CI $0.58-0.95$; $p=0.02]$ and reduction in stroke $(1.6 \%$ vs $2.7 \%$; $p=0.04)$ [48]. There was only a trend to reduced non-fatal $\mathrm{MI}(2.9 \%$ vs $3.9 \%, p=0.12)$ and no difference in $\mathrm{CV}$ death or all-cause mortality, although follow-up was relatively short at 2 years.

In summary, given their favorable CV benefits, empaglifozin, liraglutide, or possibly semaglutide should now be considered as second-line therapy in diabetic patients at high CV risk.

\section{New Lipid-Lowering Strategies}

Previous trials have demonstrated that high dose statins may be associated with reduction in coronary atherosclerosis volume as quantified by IVUS. PCSK9 (proprotein convertase subtilisin/kexin type 9) inhibitors when added to statin therapy are associated with marked further reductions in LDL-C, but it was not known if this would be associated with further reduction in atherosclerosis volume. The GLAGOV trial (Global Assessment of Plaque Regression With a PCSK9 Antibody as Measured by Intravascular Ultrasound) randomized 968 patients (baseline mean LDL-C level $2.4 \mathrm{mmol} /$ l) with angiographically moderate coronary atheroma to monthly evolocumab $420 \mathrm{mg}$ or placebo for 76 weeks in addition to moderate-high intensity statins. Evolocumab reduced LDL-C to an average of $0.94 \mathrm{mmol} / \mathrm{l}$. On repeat IVUS at 78 weeks, percentage atheroma volume (primary endpoint) was decreased by $0.95 \%$ with evolocumab vs an increase of $0.05 \%$ with placebo (between group difference 1.0\%; $p<0.001)$. Overall, plaque regression occurred in significantly more evolocumab patients $(64.3 \%$ vs $47.3 \% ; p<0.001)$ [49]. In an exploratory subgroup analysis of patients already achieving the current ESC treatment goal of LDL-C less than $1.8 \mathrm{mmol} / \mathrm{l}$, those 
receiving evolocumab achieved a mean LDL-C of $0.6 \mathrm{mmol} / \mathrm{l}$ which was associated with an even greater regression of atheroma $(-1.97 \%$ change in percentage atheroma volume and $81 \%$ of patients showing regression). Given these IVUS findings and post hoc clinical outcomes observed in phase 2 trials, results of the phase 3 outcome trial are eagerly awaited.

Patients with heterozygous familial hypercholesterolemia (HeFH) and LDL-C levels too high to be controlled by statin alone may currently be treated with serial lipoprotein apheresis which entails significant expense, patient inconvenience, and often lengthy travel to a regional lipid center. Given the marked LDL-C reduction possible with PCSK9 treatment, ODYSSEY-ESCAPE (NCT02326220) randomized HeFH patients undergoing lipoprotein apheresis in blinded fashion to alirocumab $150 \mathrm{mg}$ twice monthly vs placebo. Apheresis was deferred if LDL-C was at least 30\% lower than the baseline (pre-apheresis) value. Use of alirocumab significantly reduced requirement for apheresis with $63.4 \%$ of patients on alirocumab avoiding all and $92.7 \%$ avoiding at least half of the apheresis treatments [50]. This has significant implications for $\mathrm{HeFH}$ patients and may offer potential cost savings.

A further indication for PCSK9 inhibition may be use in patients with statin intolerance. GAUSS-3 (NCT01984424) randomized 218 patients prospectively confirmed during run in as having atorvastatin intolerance to evolocumab $420 \mathrm{mg}$ once monthly or ezetimibe $10 \mathrm{mg}$ daily. As expected, by 24 weeks evolocumab showed significantly greater reduction in LDL-C $(-52.8 \%$ vs $-16.7 \%$; $p<0.001$ ) but importantly there was no signal of excess myalgia with evolocumab; indeed the rate of discontinuation for muscle symptoms was numerically less $(0.7 \%$ vs $6.8 \%)$ [51]. Thus, PCSK9 inhibitors offer a new treatment option in selected patients with statin intolerance.

A novel approach for reducing PCSK9 activity is to inhibit liver synthesis of PCSK9 by use of small interfering RNAs (siRNAs) which selectively silence the translation of PCSK9 messenger RNA (mRNA). Inclisiran, a chemically synthesized siRNA, has been shown to produced sustained hepatocyte-specific,
PCSK9-specific RNA silencing in healthy volunteers up to 84 days after administration (Fig. 6).

Prespecified 90-day interim analysis $(n=497)$ from the phase II ORION 1 trial presented at AHA 2016 reported that a single dose of inclisiran $300 \mathrm{mg}$ achieved a mean 51\% LDL-C reduction at day 60 and 45\% LDL-C reduction by day 90 . A double $300 \mathrm{mg}$ injection strategy, the first on day 1 and the second on day 90, achieved a mean LDL-C reduction of $57 \%$ at day 120 and $52 \%$ LDL-C reduction by day 180 [52]. These results support an attractive 4-6 monthly injection dosing schedule. Complete ORION 1 results are expected in 2017 and will help guide the phase 3 clinical outcome trial programme.

While interest mounts in methods to target the PCSK9 pathway, it continues to wane regarding the cholesteryl ester transfer protein (CETP) pathway. CETP inhibition is associated with favorable effects on HDL-C, LDL-C, lipoprotein(a), and cholesterol efflux, but previous studies have failed to demonstrate reduction in MACE. Evacetrapib, a potent CETP inhibitor but which lacks off-target aldosterone effect seen with torcetrapib, was tested in the large phase III ACCELERATE (NCT01687998) trial. A total of 12,072 patients at high vascular risk were randomized with evacetrapib achieving a $130 \%$ increase in HDL-C and 37\% reduction in LDL-C vs placebo [53]. However, the trial was stopped early because of futility with no significant difference in clinical outcomes demonstrated.

\section{Multi-Risk Factor Reduction}

The importance of a multi-risk factor approach to $\mathrm{CV}$ risk reduction is well recognized. HOPE 3 (NCT00468923) aimed to determine the CV benefit of antihypertensives, rosuvastatin, or their combination in an ethnically diverse population without previous $\mathrm{CV}$ disease recruited based on overall intermediate $\mathrm{CV}$ risk rather than requiring specific BP or LDL-C levels. In total, 12,705 patients with a mean $\mathrm{BP}$ of $138 / 82 \mathrm{mmHg}$ and mean LDL-C of $3.2 \mathrm{mmHg}$ were randomized in $2 \times 2$ factorial trial design into three arms. 


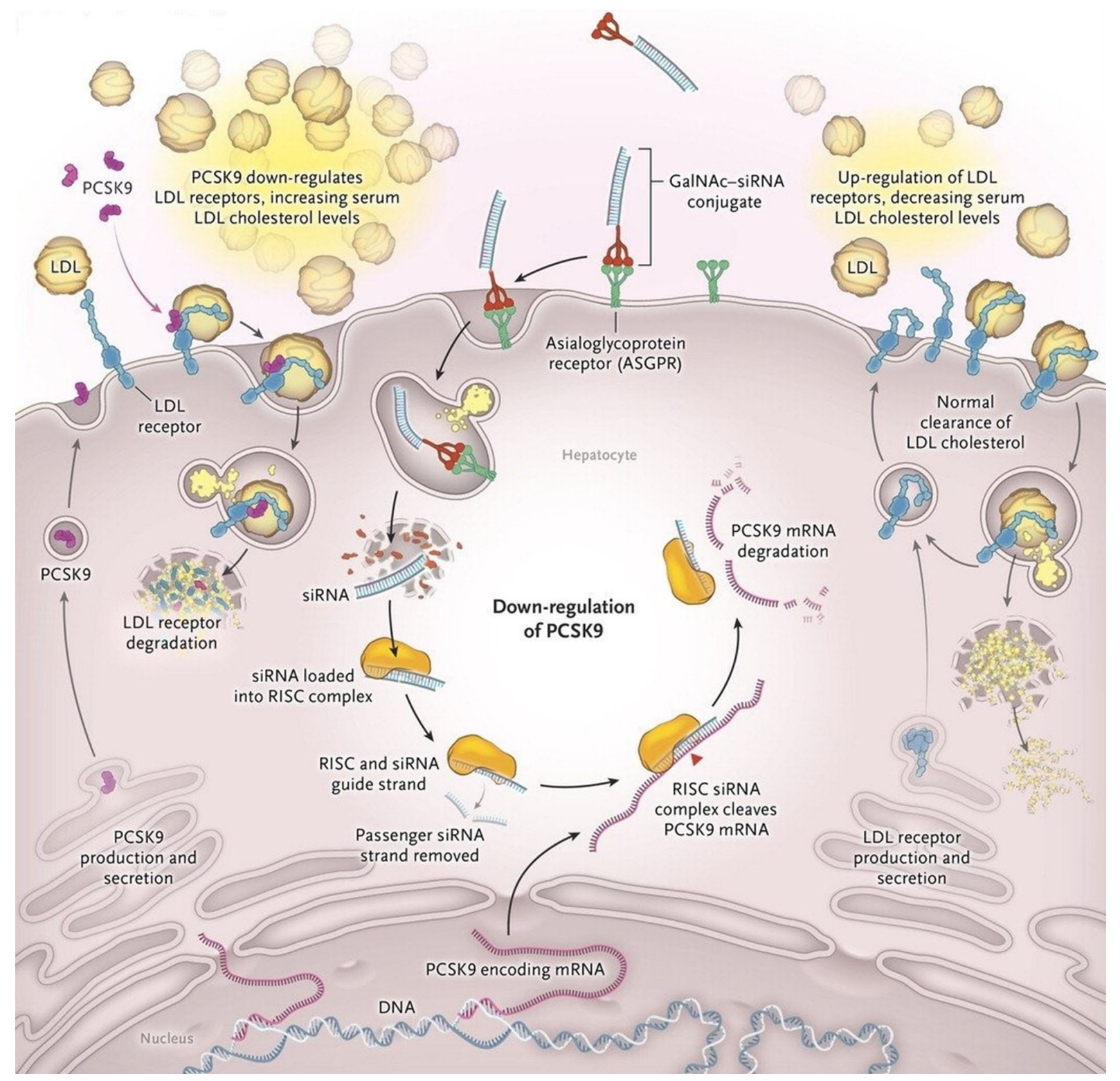

Fig. 6 Inclisiran mechanism of action: a small interfering RNA (siRNA) which selectively and catalytically silences the translation of its target PCSK9-encoding messenger
RNA (mRNA) through the formation of effector RNA-induced silencing complexes Reproduced with permission from N Engl J Med 2017; 376:4-7

0.56-0.94). Symptomatic hypotension was 1.7 times more common with antihypertensive therapy ( $3.4 \%$ vs $2.0 \% ; p<0.001)$. In the second arm, rosuvastatin $10 \mathrm{mg}$ vs placebo was associated with a $24 \%$ reduction in $\mathrm{CV}$ death, stroke, or MI (3.7\% vs $4.8 \%$, HR $0.76,95 \%$ CI $0.64-0.91$; $p=0.002)$. In the third arm rosuvastatin plus antihypertensive therapy vs placebo was 
associated with a $29 \%$ in CV death, stroke, or MI (3.6\% vs $5.0 \%$, HR $0.71,95 \%$ CI $0.56-0.9$; $p=0.005$ ) [54]. In summary, primary prevention with statin therapy in this intermediate risk population was effective, whereas antihypertensive therapy was not of benefit in the absence of elevated BP but was associated with adverse events which thus calls into question the need to routinely include antihypertensives in a polypill approach.

Cardiac rehabilitation following a CV event is associated with improved outcomes but the optimum duration is unknown. In the OPTICARE trial (NCT01395095), following ACS and a standard 3 months of cardiac rehabilitation, 914 patients were randomized to no further therapy, an additional 9 months of group therapy or an additional 9 months of telephone support. Overall, the prolonged programmes were not associated with further improvement in CV risk markers. However, compliance with the prolonged programmes was suboptimal and when only those attending more than $75 \%$ of sessions were assessed, an additional 9 months of group therapy was associated with a $37 \%$ reduction in smoking $(13.4 \%$ vs $21.3 \%$; $p<0.001)$ and a $9 \%$ reduction in total cholesterol (3.9 vs $4.3 \mathrm{mmol} / \mathrm{L} ; p<0.001$ ) [55]. This suggests that in motivated patients, a 1-year group rehabilitation programme may be of additional CV benefit. In contrast, additional telephone support was of unclear benefit.

\section{ANTIPLATELET THERAPY}

Given the increased risk of gastrointestinal (GI) hemorrhage with antiplatelet therapy, proton pump inhibitors (PPI) are often co-prescribed but there has been concern about possible interaction via CYP3A4 reducing antiplatelet efficacy particularly in high risk subgroups following PCI or ACS [56].

An updated analysis from the Clopidogrel and the optimization of gastrointestinal events trial (COGENT) (NCT00557921), which randomized patients receiving DAPT to omeprazole vs placebo, reported outcomes from PCI $(n=2676)$ and post ACS \pm PCI $(n=1573)$ subgroups [57]. In those who underwent PCI, omeprazole was associated with a 56\% reduction in GI bleed/ulcers/erosive disease at 180 days $(1.2 \%$ vs $2.7 \% ; p=0.02)$ but no clear increase in $\mathrm{CV}$ events $(5.4 \%$ vs $6.3 \%$; HR 1.00 ; 95\% CI 0.67-1.50; $p=1.00)$. In those who had an ACS \pm PCI $(n=1573)$ omeprazole was associated with a $59 \%$ reduction in GI bleed/ulcers/ erosive disease at 180 days $(1.1 \%$ vs $2.7 \%$; $p=0.05)$ but no increase in CV events $(5.6 \%$ vs $4.5 \%$; HR $1.40 ; 95 \%$ CI $0.77-2.53 ; p=0.27$ ). While the CV outcome data appear encouraging, the wide confidence limits mean it is not possible to exclude the possibility of a clinically important interaction and when combining with DAPT it may still be prudent to select PPIs associated with lower CYP3A4 interaction.

Although titration of antiplatelet dose according to platelet function testing has not been shown to improve clinical outcome in previous studies, optimizing antiplatelet anti-ischemic/bleeding balance may be more important in the elderly, given their increased risk of ischemic and bleeding events. The ANTARCTIC (Platelet function monitoring to adjust antiplatelet therapy in elderly patients stented for acute coronary syndrome) trial (NCT01538446) randomized 877 elderly patients aged 75 years and over with ACS undergoing stenting to dose-adjusted antiplatelet treatment guided by platelet function tests vs conventional treatment. Dosage increases occurred in $45 \%$ of patients in the monitoring group. However, there was no difference in the primary endpoint of CV death, MI, stroke, stent thrombosis, urgent revascularization, or bleeding complications between groups $(28 \%$ vs $28 \% ; p=0.98)$ or in rates of bleeding [58]. Thus, even in elderly patients platelet function testing did not appear useful for guiding antiplatelet dose adjustment.

Current ESC guidelines regarding P2Y12 blockers advocate the use of either the thienopyridine drug prasugrel or the cyclopentyltriazolopyrimidine drug ticagrelor in patients with acute MI. Despite their molecular differences, they display similarly fast onset and greater inhibition of platelet activity than clopidogrel and it is unclear if one has a clinical advantage over the other. The PRAGUE 18 (Prasugrel vs ticagrelor in patients with acute MI 
treated with primary percutaneous coronary intervention) trial (NCT02808767) randomized patients with acute MI to prasugrel vs ticagrelor in addition to aspirin therapy. The trial was discontinued prematurely after only 1230 of the planned 2500 patients were enrolled on the basis of futility due to a lack of significant difference in the primary endpoint of death, reinfarction, urgent TVR, stroke, or serious bleeding [59]. While results support continued use of both agents, the study was underpowered to draw firm conclusions.

Ticagrelor has previously been shown to be superior to clopidogrel for reducing cardiac events but it was unclear if its benefits extended to peripheral arterial disease. The EUCLID trial (Examining use of ticagrelor in peripheral artery disease; NCT01732822) randomized 13,885 patients with symptomatic peripheral artery disease to receive either ticagrelor $(90 \mathrm{mg}$ twice daily) or clopidogrel (75 mg daily) [60]. Unlike in the ACS setting, ticagrelor was not superior to clopidogrel with no difference in the primary efficacy endpoint of $\mathrm{CV}$ death, MI, ischemic stroke $(10.8 \%$ vs $10.6 \%)$, acute limb ischemia $(1.7 \%$ vs $1.7 \%)$, or major bleeding $(1.6 \%$ vs $1.6 \%)$. Thus EUCLID does not support any change to the current management of these patients.

\section{ADVANCES IN HEART FAILURE}

Neurohormonal modulation is central to contemporary heart failure management. Hyperkalemia may be a more frequent concern when combining mineralocorticoid receptor antagonists (MRA) with renin-angiotensin-aldosterone inhibitors such as ACE inhibitors or angiotensin receptor/neprilysin inhibitors (ARNI). Within the PARADIGM-HF trial (Prospective Comparison of ARNI With an ACE Inhibitor to Determine Impact on Global Mortality and Morbidity in Heart Failure; NCT01035255) 4671 patients were receiving an MRA at baseline [61]. The combination of MRA plus ARNI vs MRA plus ACE inhibitor was associated with similar incidence of any hyperkalemia (potassium $>5.5 \mathrm{mmol} / \mathrm{L}$ ) but a lower incidence of severe hyperkalemia (potassium
$>6.0 \mathrm{mmol} / \mathrm{L})$ (2.2 vs 3.1 per 100 patient-years; $p=0.02$ ). In patients who newly started taking MRAs during the trial, severe hyperkalemia was also less common in those combining MRA with ARNI rather than ACE inhibitor (2.3 vs 3.3 per 100 patient-years; $p=0.003$ ) suggesting neprilysin inhibition may facilitate safer use of MRAs in patients at risk of hyperkalemia.

The direct renin inhibitor aliskiren represents one of the most recent targets for renin-angiotensin system blockade. The ATMOSPHERE trial (Aliskiren Trial to Minimize Outcomes in Patients with Heart Failure; NCT00853658) randomized 7016 patients with stable NYHA class II-III heart failure, LVEF less than $35 \%$, and raised $\mathrm{BNP} / \mathrm{NT}$ pro-BNP to one of three groups: aliskiren $300 \mathrm{mg}$ once daily or aliskiren plus enalapril $5-10 \mathrm{mg}$ twice daily combination therapy vs enalapril alone (standard care). As a result of later safety concerns regarding aliskiren use in diabetic patients, the trial was amended to exclude diabetics. At a mean of 36.6 months follow-up, neither aliskiren monotherapy nor aliskiren/enalapril combination therapy was superior to enalapril alone for the primary endpoint of $\mathrm{CV}$ death or hospitalization for heart failure (33.8\% vs $32.9 \%$ vs $34.6 \%)$. However, combination therapy was associated with a higher incidence of increased creatinine or potassium and of hypotension [62]. Thus, given previous trial data, aliskiren remains primarily reserved as an option for hypertension.

MRAs have been extensively studied in chronic heart failure, but less so in acute heart failure. In patients with acute heart failure, intravenous loop diuretics are central to usual care but their use intensifies secondary hyperaldosteronism, enhancing proximal tubular sodium absorption and decreased distal sodium delivery, thereby contributing to diuretic resistance. While low doses of MRAs used in chronic HF are believed to benefit patients primarily by antifibrotic effects, higher doses of MRAs may cause significant natriuresis. The ATHENA-HF trial (Aldosterone Targeted NeuroHormonal Combined with Natriuresis therapy in Heart failure; NCT02235077) randomized 360 patients hospitalized with acute heart failure to high dose spironolactone $100 \mathrm{mg}$ od for $96 \mathrm{~h}$ vs 
placebo in addition to standard care. Although high dose spironolactone was well tolerated, no significant difference in reduction of NT-proBNP was observed ( -0.55 vs -0.49 ; $p=0.57$ ) [63]. Whether benefit would be seen by using higher spironolactone doses or by targeting patients with more marked fluid retention remains unclear.

In addition to renin-angiotensin system approaches, modulation of autonomic nervous system imbalance is central to contemporary heart failure management. Previous small studies have reported possible positive benefits of vagal nerve stimulation on ECHO reverse remodeling and quality of life parameters. The INOVATE-HF trial (Increase of vagal tone in congestive heart failure; NCT01303718) randomized 707 patients with heart failure (NYHA III, $\mathrm{EF}<40 \%)$ to device-mediated vagal nerve stimulation (Biocontrol Cardiofit ${ }^{\circledR}$, Biocontrol Medical, Yehud, Israel) (Fig. 7) vs medical therapy. Disappointingly, at 16 months, vagal nerve stimulation vs placebo showed no difference in the primary endpoint of death or first event heart failure $(25.8 \%$ vs $30.3 \% ; p=0.37)$ and although improvements in NYHA symptom class, quality of life, and 6-min walk test $(p<0.05)$ were reported, there was no difference in LV end-systolic volume index by ECHO [64].

Invasive monitoring of pulmonary artery pressure using permanently implanted devices has been shown to reduce acute heart failure

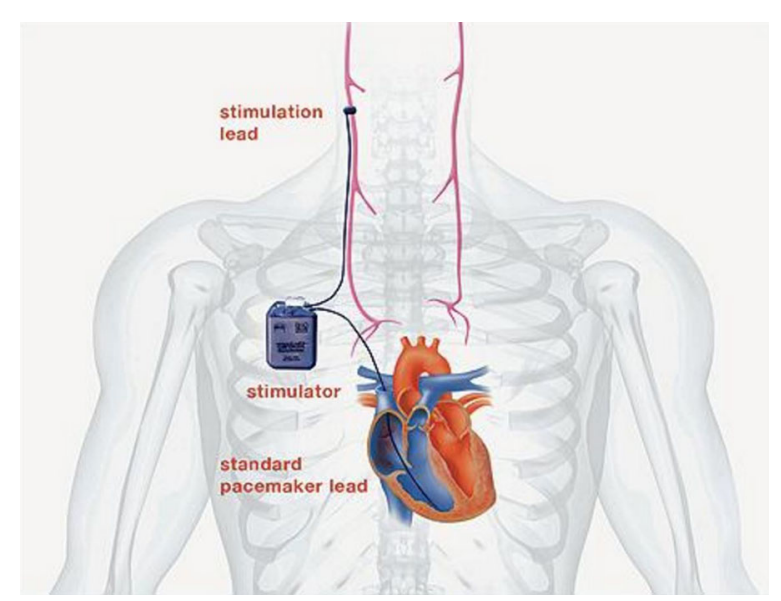

Fig. 7 Biocontrol Cardiofit ${ }^{\circledR}$. Reproduced with permission Biocontrol Medical, Yehud, Israel admissions [65]. The clinical usefulness of non-invasive lung impedance measurement (as a marker for lung fluid content) was assessed in the IMPEDANCE-HF trial (Non-Invasive Lung IMPEDANCE-Guided Pre-emptive Treatment in Chronic Heart Failure Patients; NCT101315223) which randomized 256 with heart failure (NHYA II-IV, EF <35\%) to management guided by non-invasive lung impedance (LI) measurement plus clinical assessment vs clinical assessment alone. Use of LI was associated with increased medication adjustment, a marked reduction in acute heart failure admissions (67 vs $158, p<0.001$ at 1 year; 211 vs $386, p<0.001$ at study end), and reduction in all-cause mortality [66]. Importantly, decreases in LI appeared to precede hospitalizations. While the LI device needs further study and comparison with other implantable hemodynamic monitoring devices, IMPEDANCE HF supports the emerging evidence base that early detection of subtle hemodynamic deteriorations in heart failure in the outpatient setting may improve both clinical outcomes and cost-effectiveness of heart failure management.

By contrast, the REM-HF trial (Remote Management of Heart Failure Using Implantable Devices; ISRCTN96536028) [67], which randomized 650 heart failure patients with CRT-P, CRT-D, and implantable cardioverter defibrillator (ICD) devices to weekly remote data downloads vs usual care (36 monthly clinic data downloads), failed to demonstrate any reduction in all-cause mortality or acute hospital admissions despite $70 \%$ of patients in the weekly download group having alterations to treatment plans.

Current American and European guidelines support the implantation of a prophylactic cardio-defibrillator for patients with severe LV systolic dysfunction. While survival benefit has clearly been demonstrated in patients with ischemic heart failure it is less clear whether this extends to the non-ischemic population. The Defibrillator implantation in patients with non-ischemic systolic heart failure (DANISH) study (NCT00542945) randomized 1116 patients with non-ischemic systolic heart failure (EF $<35 \%)$ to ICD vs medical therapy alone. Use of ICD was not associated with a reduction in 
the primary outcome of all-cause mortality at a mean of 67.6 months $(21.6 \%$ vs $23.4 \%$; $p=0.28)$ and there was no difference in $\mathrm{CV}$ death $(13.8 \%$ vs $17 \% ; \quad p=0.1, \quad 95 \% \quad$ CI 0.57-1.05), although the incidence of sudden cardiac death was halved $(4.3 \%$ vs $8.2 \%$; $p=0.005,95 \%$ CI 0.31-0.82) [68]. As the results of this trial are at odds with the previously published SCD-HeFT [69] and DEFINITE [70] trials, further studies within non-ischemic heart failure patients are required to help clarify those who benefit or otherwise from ICD.

An evolving field in heart failure research involves the use of cardiopoietic stem cells. Isolation of mesenchymal stem cells from a patient's own bone marrow may be exposed to a cardiogenic cocktail turning them into cardiopoietic cells which can then be injected into damaged heart tissue. The CHART-1 trial (Congestive Heart Failure Cardiopoietic Regenerative Therapy; NCT01768702) study randomized 271 patients with ischemic cardiomyopathy (LVEF $\leq 35 \%$ ) to stem cell injection $(n=120)$ vs a sham procedure [71]. At 39 weeks, there was no difference in the primary efficacy endpoint (death, worsening heart failure, quality of life score, 6-min walk distance, LV end-systolic volume and LVEF). However, stem cell injection in the subgroup with baseline LV dilatation (LV end-diastolic volume 200-370 mL) was associated with benefit $(p=0.015)$ and a lower injection intensity also appeared helpful; these findings will help shape the design of the CHART-2 US study.

Iron deficiency is present in approximately $50 \%$ of patients with chronic heart failure which may lead to reduced hemoglobin and to impaired oxygen utilization at a tissue level. IV administration of iron has previously been shown to improve NYHA status (FAIR-HF) and 6-min walk (CONFIRM-HF) [72]. The effect on peak VO2 was assessed in EFFECT-HF (Effect of Ferric Carboxymaltose on Exercise Capacity in Patients With Iron Deficiency and Chronic Heart Failure; NCT01394562), which randomized 174 patients with stable heart failure and iron deficiency to IV administration of ferric carboxymaltose vs standard care. At 24 weeks, IV administration of iron was associated with a marked increase in ferritin and mean peak VO2 improved by $1.04 \pm 0.44 \mathrm{~mL} / \mathrm{kg} / \mathrm{min}$ (95\% CI 0.16-1.91) vs standard care [73]. These phase II trials are highly encouraging and larger trials are now planned to assess the effect of IV administration of iron on clinical outcomes.

However, regular IV administration of iron poses logistical challenges and is expensive; hence, oral administration of iron has been used by some clinicians as a substitute. The IRONOUT HF trial (Oral Iron Repletion effect ON oxygen Uptake in Heart Failure) trial (NCT02188784) randomized 225 patients with stable heart failure to oral administration of iron polysaccharide (150 mg twice daily) vs placebo. Unfortunately, oral administration of iron only increased ferritin by $11 \%$ (in keeping its known poor absorption in heart failure patients) and at 16 weeks there was no improvement in exercise capacity [74]. Although the trial duration was short, the minimal response suggests that regular oral administration of iron is unlikely to be effective.

Whilst much progress has been made over the last two decades in chronic management of heart failure patients, there have been limited advances in the acute setting. It has been suggested that early treatment during acute decompensation might reduce acute LV distension and thus reduce myocardial micro-injury and potentially improve later clinical outcomes. TRUE-AHF (Trial of Ularitide Efficacy and Safety in Heart Failure; NCT01661634) randomized 2157 patients with acute heart failure to early (less than $12 \mathrm{~h}$ ) treatment with the synthetic natriuretic peptide ularitide vs placebo. In keeping with previous vasodilator trials, ularitide vs placebo was associated with a $47 \%$ reduction in BNP and reduction in early heart failure events ( 55 vs $87 ; p=0.005$ ). However, this was not associated with any difference in troponin release or longer-term clinical outcome [75]. Thus, the current role of vasodilator therapy therefore appears to be confined to symptomatic relief in acute heart failure.

Previous 5-year data from the STICH trial (Surgical Treatment for Ischemic Heart Failure) failed to demonstrate a mortality benefit for CABG among 1212 patients with severe ischemic cardiomyopathy (LVEF <35\%) randomized to CABG plus medical therapy (610 
patients) vs medical therapy alone (602 patients). However, long-term data at a median follow-up of 9.8 years (range 3.5-13.4) presented at the ACC (from 98\% of patients) reported a $16 \%$ reduction in the primary outcome of all-cause mortality vs medical therapy (58.9\% vs $66.1 \%$; HR 0.84 , CI $0.73-0.97$; $p=0.02$ ) and median survival was 7.73 years in the CABG group vs 6.29 years in the medical therapy group. Thus if clinically appropriate, CABG remains preferable to conservative therapy for complex coronary disease and ischemic cardiomyopathy [76].

\section{ADVANCES IN ARRHYTHMIA AND ELECTROPHYSIOLOGY}

\section{Atrial Fibrillation Detection}

ESC guidelines recommend opportunistic screening for AF in patients over 65 (level 1B) by means such as pulse assessment or rhythm strip analysis, although it is recognized that this may fail to detect many paroxysmal episodes of subclinical AF. Previous trials in patients with pacemakers or ICDs have shown that subclinical AF (atrial high rate episodes) is common and is associated with increased incidence of subsequent stroke but the prevalence in patients not requiring a device has been unknown. The ASSERT II trial (Prevalence of Sub-Clinical Atrial Fibrillation Using an Implantable Cardiac Monitor; NCT01694394) recruited 256 patients aged 65 years and over with at least one clinical risk factor (CHADS2VASC $\geq 2$, obstructive sleep apnea, or BMI $>30$ ) and one echo or biochemical risk factor (left atrial diameter $\geq 4.4 \mathrm{~cm}$, left volume $\geq 58 \mathrm{~mL}$, or NT-ProBNP $\geq 290 \mathrm{pg} / \mathrm{ml}$ ). All patients received an implantable loop monitor (Confirm DM2100; St Jude Medical) for between 9 and 18 months. The annualized incidence of subclinical AF of at least 5 min was common-observed in $34.4 \%$ of patients per year-and was almost twice as common in patients with left atrial volume greater than $73.5 \mathrm{~mL}(51.9 \%$ vs $27.4 \% ; p=0.015)$ but, of interest, it was not any more common in the fifth of patients with a prior history of stroke [77]. Thus, it remains unclear if all subclinical
AF patients require anticoagulation, and further studies are investigating this key question including ARTESiA (Apixaban for the Reduction of Thrombo-Embolism in Patients With Device-Detected Sub-Clinical Atrial Fibrillation; NCT01938248) and NOAH (Non-vitamin K Antagonist Oral Anticoagulants in Patients With Atrial High Rate Episodes; NCT02618577).

\section{Non-Vitamin K Antagonists (NOACs)}

The use of non-vitamin $\mathrm{K}$ antagonist oral anticoagulants (NOAC) has revolutionized anticoagulation in atrial fibrillation. However, many concerns still exist over the place of these agents following major bleeding, PCI, and in planned cardioversion. Several trials presented in 2016 aimed to address these specific areas.

Despite landmark clinical trial data, the previous lack of specific NOAC reversal agents posed concern for some clinicians. Idarucizumab is now approved for reversal of dabigatran [25]. Andexanet, a factor Xa decoy protein, has previously been shown, among healthy volunteers, to reduce anti-Xa activity and to restore thrombin generation. ANNEXA-4 (Andexanet Alfa for Acute Major Bleeding Associated with Factor Xa Inhibitors; NCT02329327) evaluated 67 patients who had acute major bleeding within $18 \mathrm{~h}$ after the administration of a factor Xa inhibitor (rivaroxaban, apixaban, or enoxaparin) [78]. All received a 30-min bolus followed by a 2 -h infusion of adexanet. Dose was determined on timing of last administration of factor Xa inhibitor. After the bolus dose median anti-factor Xa activity decreased by $89-93 \%$ (with similar suppression maintained during the 2-h infusion). By $4 \mathrm{~h}$ after the infusion there was partial recovery of anti-factor Xa activity (30-39\% reduction from baseline) but by $12 \mathrm{~h}, 79 \%$ of patients had achieved clinical hemostasis (Fig. 8).

Of note, thrombotic events occurred in 12 patients and 10 died (six due to cardiac cause) during 30-day follow-up. Overall, andexanet appears a useful option although given the short plasma half-lives of the anticoagulants studied, the relatively long median 4.8-h delay from presentation to the administration of andexanet, and the absence of a placebo arm, it 


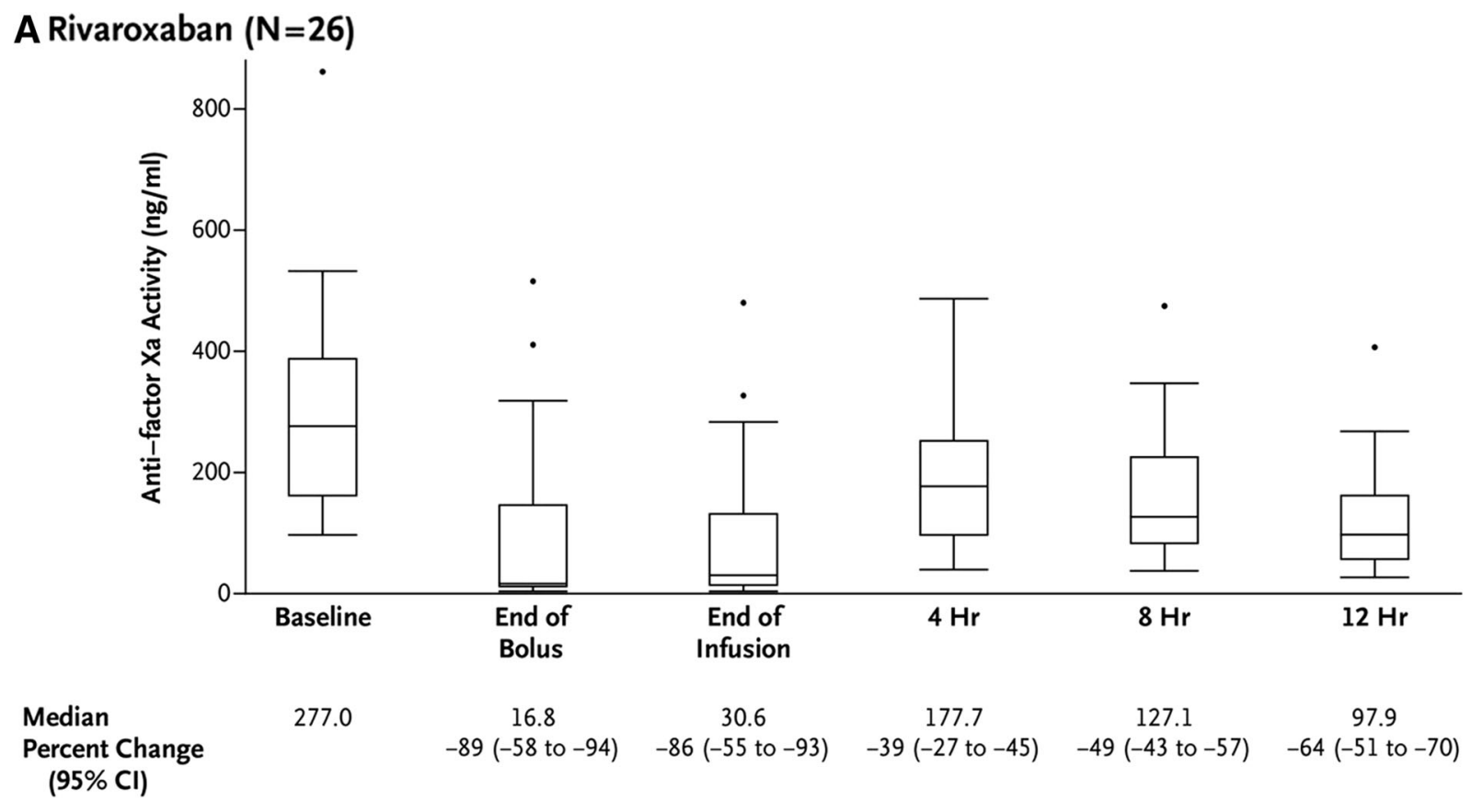

\section{B Apixaban ( $\mathbf{N}=\mathbf{2 0})$}

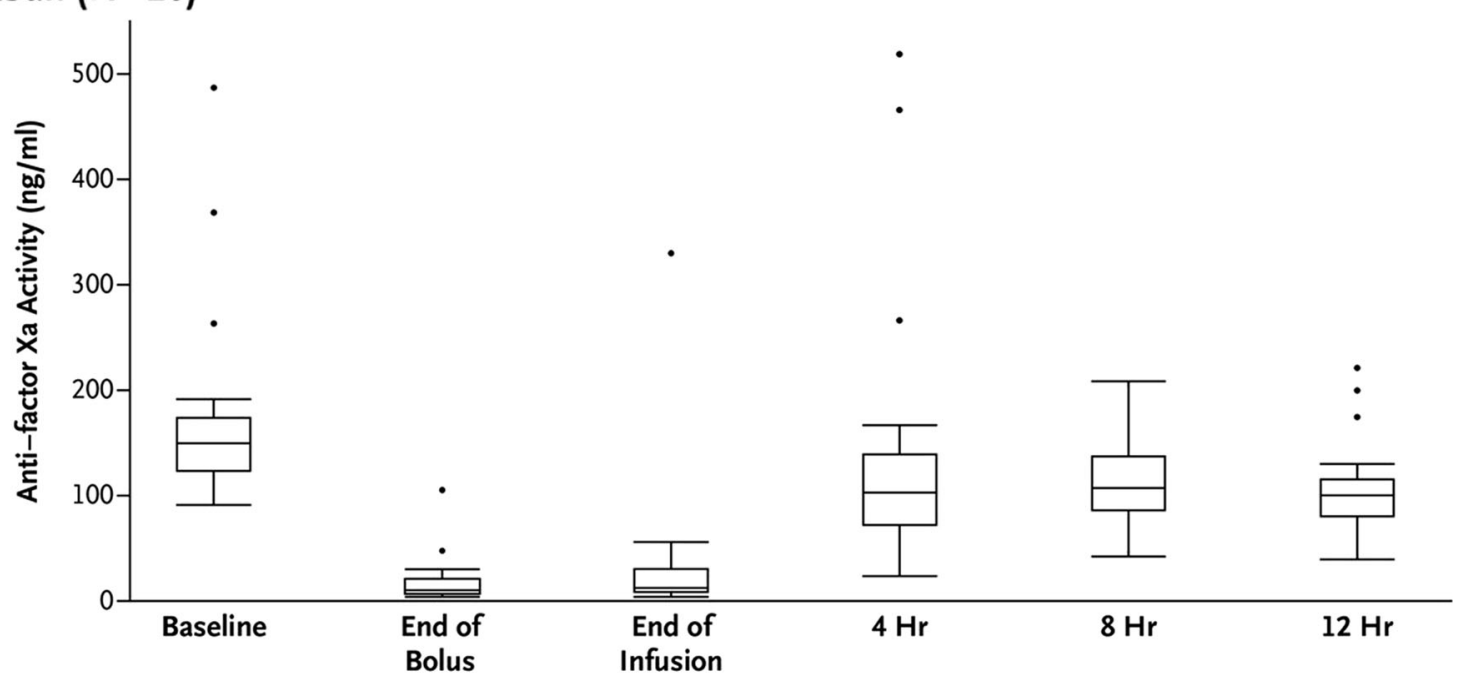

$\begin{array}{llccccc}\text { Median } & 149.7 & 10.3 & 12.5 & 103.0 & 107.1 & 100.2 \\ \begin{array}{l}\text { Percent Change } \\ (95 \% \mathrm{Cl})\end{array} & & -93(-87 \text { to }-94) & -92(-85 \text { to }-94) & -30(-23 \text { to }-46) & -28(-19 \text { to }-38) & -31(-27 \text { to }-41)\end{array}$

Fig. 8 Anti-factor Xa activity following Andexanet Alfa. Reproduced with permission from [78]

cannot be determined how many patients would have achieved hemostatic efficacy without andexanet. Further refinements in the administration of andexanet could lead to more complete reversal of anti-factor Xa activity and improved clinical outcomes.
An ongoing important area of uncertainty relates to patients with AF undergoing PCI. In the absence of definitive trial data, current ESC revascularization guidelines for non-ST elevation MI [79] suggest different treatment strategy options: (class IIa) triple therapy (anticoagulant, 
aspirin plus clopidogrel) for 6 months in low-bleeding-risk patients or for 1 month in high-bleeding-risk patients and (class IIb) anticogulation plus clopidogrel only as an alternative to triple therapy in selected patients (based on WOEST study data).

PIONEER-AF (Patients with atrial fibrillation undergoing coronary stent placement; NCT01830543) is the first study to compare a NOAC with warfarin in this setting and randomized 2124 patients (Fig. 9) to (group 1, "WOEST-like") rivaroxaban $15 \mathrm{mg}$ once daily with a single P2Y12 inhibitor for 12 months; (group 2, "ATLAS ACS 2-like") rivaroxaban $2.5 \mathrm{mg}$ twice daily with DAPT for 1,6 , or 12 months at the operators discretion then, as needed, rivaroxaban $15 \mathrm{mg}$ once daily plus aspirin until 12 months; or (group 3, "triple therapy") warfarin with DAPT for 1,6 , or 12 months at the operator's discretion then, as needed, warfarin plus aspirin until 12 months [80]. DAPT duration was 1 month in $16 \%$, 6 months in $35 \%$, and 12 months in $49 \%$. Clopidogrel was the P2Y12 inhibitor used in $95 \%$ of cases. Clinically relevant bleeding was reduced in patients receiving rivaroxaban vs warfarin (group 1, 16.8\% and group 2, 18\% vs group $3,26.7 \% ; p<0.001$ for both). TIMI major bleeding was numerically less but not significant $(2.1 \%$ vs $1.9 \%$ vs $3.3 \%)$. Stent thrombosis rates were similar $(0.8 \%$ vs $0.9 \%$ vs $0.7 \%$ ) and were equivalent between groups. Although the composite of $\mathrm{CV}$ death, MI, or stroke was similar between groups $(6.5 \%, 5.6 \%$ vs $6.0 \%$ ) the study was not powered to test efficacy and wide confidence limits could not exclude an excess of events with rivaroxaban. Reduced all-cause re-hospitalization with rivaroxaban was described in post hoc analysis.

It would be helpful to have further trials powered for efficacy and to include a warfarin "WOEST arm" (warfarin plus clopidogrel for 12 months since this was associated with reduced MACE in WOEST). However, given the reduction in bleeding and convenience of a simple double therapy for 12 months rather than triple therapy with stepdown to double, rivaroxaban $15 \mathrm{mg}$ once a day plus single antiplatelet therapy may become the approach of choice once approved.

Following a major bleeding event clinicians are faced with the dilemma of whether to recommence anticoagulants. A recent US observational study has suggested improved outcomes in those who are restarted on anticoagulants following a bleeding event. A large Danish registry study identified 2662 patients with AF who had suffered a hemorrhagic stroke

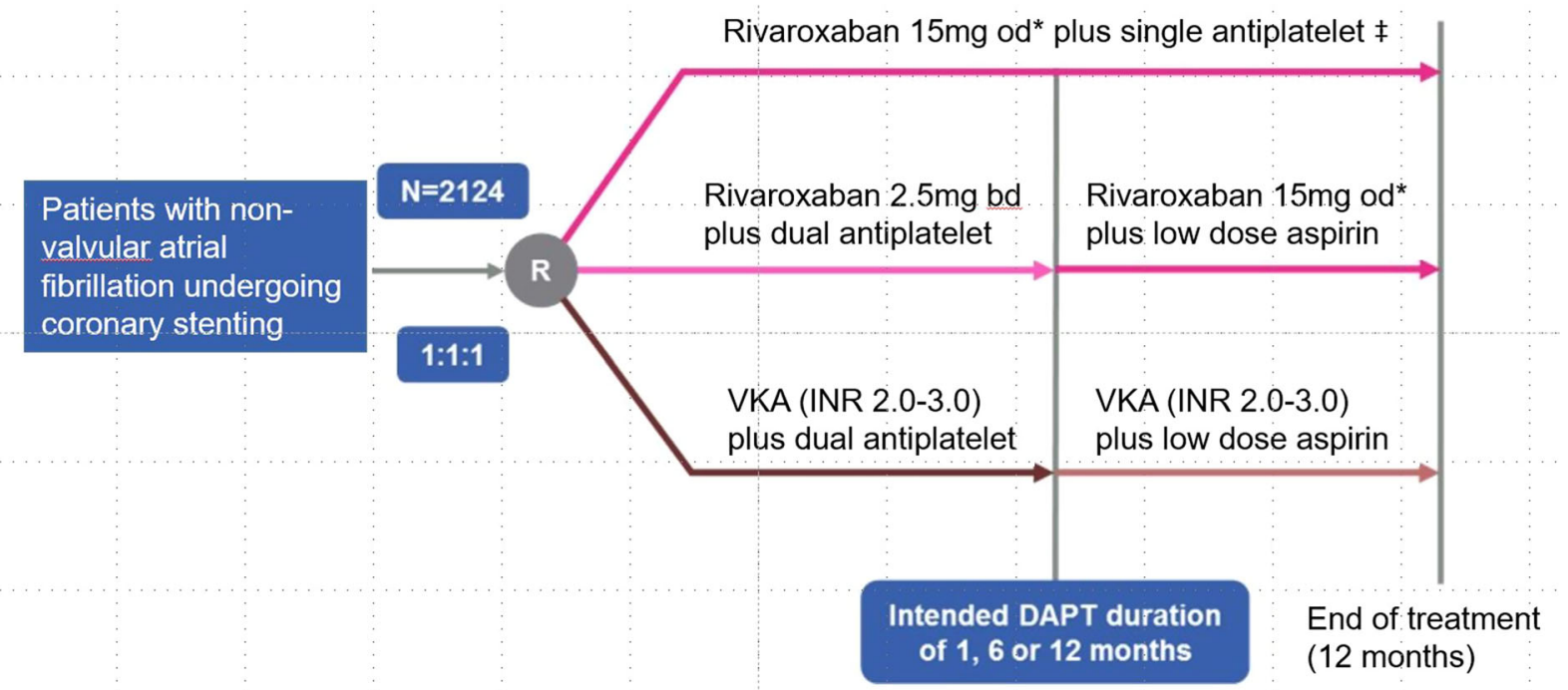

Fig. 9 PIONEER AF trial design. *If creatine clearance $30-49 \mathrm{~mL} / \mathrm{min}$ dose reduced to $10 \mathrm{mg}$ od; ${ }^{\ddagger}$ single antiplatelet $=$ clopidogrel $85 \%$, prasugrel, or ticagrelor; $V K A$ vitamin $\mathrm{K}$ antagonist, $I N R$ international normalized ratio 
or traumatic intracranial bleed while on anticoagulants [81]. In those that restarted oral anticoagulants there was an overall reduction in all-cause mortality at a mean of 2.1 years follow-up. In those with hemorrhagic stroke there was a non-significant trend towards increased risk of recurrent intracranial hemorrhage but no signal was seen for those with prior traumatic intracranial bleed. Findings thus support reinitiation of anticoagulants following intracranial hemorrhage but further work is required to determine optimum timing of reinitiation and patient risk stratification.

The use of NOACs in place of warfarin to support cardioversion has, to date, been based on retrospective analysis of the general AF trials and a smaller prospective study with rivaroxaban. ENSURE-AF (Edoxaban vs enoxaparin-warfarin in patients undergoing cardioversion of atrial fibrillation; NCT02072434), the largest prospective trial to compare a NOAC with optimized warfarinization (including bridging enoxaparin until INR was therapeutic for more than 2 days) randomized 2199 patients to edoxaban vs warfarin. The warfarin group received optimized treatment. There was a non-significant numerical reduction in the composite of $\mathrm{CV}$ death MI, stroke, embolism, or major bleeding $(0.7 \%$ vs $1.4 \%$; OR 0.50, 95\% CI 0.19-1.25) although ENSURE AF was not powered to fully test these outcomes [82]. Edoxaban appeared similarly useful whether cardioversion was undertaken after a conventional 3-week course of anticoagulation or transesphageal echocardiogram-guided cardioversion within a few days. Overall, this study adds to previous trial data suggesting that a NOACs-supported approach appears an effective alternative to conventional VKA-based anticoagulation for pericardioversion use.

\section{AF Ablation}

Pulmonary vein isolation using radiofrequency (RF) ablation has become an established treatment for AF refractory to pharmacological management, although the previous complexity and duration of the procedure have limited its availability. An alternative method using cryogenic energy delivered via balloon (cryoballoon) offers a significantly reduced procedural time. The FIRE AND ICE trial (NCT01490814) randomized 762 patients to cryoballoon vs RF ablation. Cryotherapy was associated with shorter procedural time (124 vs $14 \mathrm{~min} ; p<0.001$ ) [83]. At a mean of 1.5 years, there was no difference in the primary endpoint of recurrence of atrial arrhythmia, use of anti-arrhythmic agent, or repeat procedure (34.6\% with cryoballoon cohort vs $35.9 \%$ with RF, CI $0.76-1.22 ; p<0.001$ for non-inferiority). Phrenic nerve injury was seen in $2.7 \%$ of cryoballoon cases, although all but one resolved by 12 months. This trial further supports the use of cryoballoon ablation as an option for AF ablation. The second-generation cryoballoon has also shown improvement in long-term pulmonary vein isolation likely attributable to the extensive wide-area circumferential ablation that is achieved.

\section{CONCLUSION}

In this paper we have highlighted and summarized numerous important studies in the field of cardiology which have been published and/or presented to major international cardiology meetings throughout 2016. Many of these studies will contribute to updating of current practice guidelines and others will play an integral role in the advancement and development of new therapeutic strategies.

\section{ACKNOWLEDGEMENTS}

No funding or sponsorship was received for this study or publication of this article. All named authors meet the International Committee of Medical Journal Editors (ICMJE) criteria for authorship for this manuscript, take responsibility for the integrity of the work as a whole, and have given final approval for the version to be published.

Disclosures. Ian B. A. Menown reports grants to institution, honoraria and/or 
conference sponsorship from Biosensors, Boston Scientific, Sanofi Aventis, Meril Life, Menarini, Orbus Neich, Astra Zeneca, Bayer, Boehringer Ingelheim, Daichii Sankyo, Lilly, Bristol Myers Squibb, and Pfizer. Alastair Gray and Conor McQuillan have nothing to disclose.

Compliance with Ethics Guidelines. This article is based on previously conducted studies and does not involve any new studies of human or animal subjects performed by any of the authors.

Data Availability. Data sharing is not applicable to this article as no new datasets were generated or analyzed during the current study.

Open Access. This article is distributed under the terms of the Creative Commons Attribution-NonCommercial 4.0 International License (http://creativecommons.org/licenses/ by-nc/4.0/), which permits any noncommercial use, distribution, and reproduction in any medium, provided you give appropriate credit to the original author(s) and the source, provide a link to the Creative Commons license, and indicate if changes were made.

\section{REFERENCES}

1. Kelbaek H, Hofsten DE, Kober L, et al. Deferred versus conventional stent implantation in patients with ST-segment elevation myocardial infarction (DANAMI 3-DEFER): an open-label, randomised controlled trial. Lancet. 2016;387(10034):2199-206.

2. Connolly M, McEneaney D, Morgan N, Menown IBA, Harbinson M. Novel biomarkers of acute kidney injury following contrast coronary angiography. Cardiol Rev. 2015;23(5):240-6.

3. Bhatt DL. Safety and efficacy of CMX-2043 for periprocedural injury protection in subjects undergoing coronary angiography at risk of radio-contrast induced nephropathy-CARIN. Presented at American College of Cardiology Scientific Session. April 2-4, 2016, Chicago, IL.

4. Pan M, Ojeda S, Villanueva E, et al. Structural damage of jailed guidewire during the treatment of coronary bifurcations: a microscopic randomised trial. EuroPCR 2016, May 18, 2016, Paris, France.
5. Zwaan E. Upper extremity functional post-transradial PCI: interim results. EuroPCR 2016. May 18, 2016, Paris, France.

6. Jeger RV, Farah A, Engstrøm T, et al. Drug-eluting vs bare metal stents in saphenous vein grafts: the prospective randomized BASKET-SAVAGE trial. ESC Congress, August 30, 2016, Rome, Italy.

7. Menown IBA, Noad R, Garcia EJ, Meredith I. The platinum chromium element stent platform: from alloy, to design, to clinical practice. Adv Ther. 2010;27(3):129-41.

8. Bønaa KH, Mannsverk J, Wiseth $\mathrm{R}$, et al. Drug-eluting or bare-metal stents for coronary artery disease. N Eng J Med. 2016;375(16):1542-52.

9. Kaiser C, Galatius S, Erne P, et al. Drug-eluting versus bare-metal stents in large coronary arteries. N Eng J Med. 2010;363:2310-9.

10. Mauri L, Kereiakes DJ, Yeh RW, et al. Twelve or 30 months of dual antiplatelet therapy after drug-eluting stents. N Eng J Med. 2014;371:2155-66.

11. Nakamura $M$, et al. 6 months versus 18 months dual antiplatelet treatment for patients underwent bioabsorbable polymer and abluminal coated DES deployment: NIPPON randomized study. ESC Congress; August 28, 2016, Rome, Italy.

12. Lassen JF, Holm NR, Banning A, et al. Percutaneous coronary intervention for coronary bifurcation disease: 11th consensus document from the European Bifurcation Club. EuroIntervention 2016;12(1):38-46.

13. Ferenc M, Gick M, Comberg T. Culotte stenting vs TAP stenting for treatment of de-novo coronary bifurcation lesions with the need for side-branch stenting: the Bifurcations Bad Krozingen (BBK) II angiographic trial. Eur Heart J. 2016;37(45): $3399-405$.

14. Erghlis A, Kumsars I, et al. Randomised comparison of coronary bifurcation stenting with the crush versus the culotte technique using Sirolimus eluting stents. Circ Cardiovasc Interv. 2009;2:27-34.

15. Pasupathy S. NACIAM trial: The early use of $\mathrm{N}$-acetylcysteine (NAC) with glyceryl trinitrate (GTN) in ST-segment elevation myocardial infarction patients undergoing primary percutaneous coronary intervention. European Society of Cardiology 2016 Congress, August 28, 2016, Rome, Italy.

16. Chang HJ, Achenbach S. ESC 2016 Late-Breaking Clinical Trial: CONSERVE: direct catheterization versus selective catheterization guided by coronary computed tomography in patients with stable suspected coronary artery disease. European Society of Cardiology, August 29, 2016, Rome, Italy. 
17. Greenwood JP, Ripley DP, Berry C. Effect of care guided by cardiovascular magnetic resonance, myocardial perfusion scintigraphy, or NICE guidelines on subsequent unnecessary angiographic rates: the CE-MARC 2 randomized clinical trial. JAMA. 2016;316(10):1051-60.

18. The SCOT-Heart investigators. CT coronary angiography in patients with suspected angina due to coronary heart disease (SCOT-HEART): an open-label, parallel-group, multicentre trial. Lancet. 2015;385(9985):2383-91.

19. Greenwood JP, Maredia N. Cardiovascular magnetic resonance and single-photon emission computed tomography for diagnosis of coronary heart disease (CE-MARC): a prospective trial. Lancet. 2012;379:453-60.

20. Stone GW, Sabik JF, Serruys PW, et al. Everolimus-eluting stents or bypass surgery for left main coronary artery disease. $N$ Eng J Med. 2016;375:2223-35.

21. Mäkikallio T, Holm NR, Lindsay M, Spence MS, Erglis A, Menown IB, et al. Percutaneous coronary angioplasty versus coronary artery bypass grafting in treatment of unprotected left main stenosis (NOBLE): a prospective, randomized, open-label, non-inferiority trial. Lancet. 2016;388(10061):2743-52.

22. Morice MC, et al. Five-year outcomes in patients with left main disease treated with either percutaneous coronary intervention or coronary artery bypass grafting in the synergy between percutaneous coronary intervention with taxus and cardiac surgery trial. Circulation. 2014;129:2388-94.

23. Menown IBA, on behalf of the NOBLE investigators. Syntax stratified NOBLE results. Presented at Transcatheter Cardiovascular Therapeutics, 2016.

24. Taggart DP, Altman DG, Gray AM, et al. For the ART Investigators Randomized trial of bilateral versus single internal-thoracic-artery grafts. N Eng J Med. 2016;375:2540-9.

25. McCune $C$, McKavanagh $P$, Menown IBA. A review of the key clinical trials of 2015: results and implications. Cardiol Ther. 2016;5(2):109-32.

26. Garot P, Morice MC, Tresukosol D, Pocock S, Meredith I, Abizaid A, Carrié D, Naber C, Iñiguez A, Talwar S, Menown IBA, et al. Two-year outcomes of high bleeding risk patients after drug-coated stents. J Amer Coll Cardiol. 2017;69(2):162-71.

27. Serruys PW, Farooq V, Kalesan B, et al. Improved safety and reduction in stent thrombosis associated with biodegradable polymer-based biolimus-eluting stents versus durable polymer-based sirolimus-eluting stents in patients with coronary artery disease: final 5-year report of the LEADERS (Limus Eluted From A Durable Versus ERodable Stent Coating) randomized, noninferiority trial. JACC Cardiovasc Interv. 2013;6(8): 777-89.

28. Pilgrim T, Heg D, Roffi $M$, Tüller $\mathrm{D}$, Muller $\mathrm{O}$, Vuilliomenet A, et al. Ultrathin strut biodegradable polymer sirolimus-eluting stent versus durable polymer everolimus-eluting stent for percutaneous coronary revascularisation (BIOSCIENCE): a randomised, single-blind, non-inferiority trial. Lancet. 2014;384(9960):2111-22.

29. Teeuwen K, van der Schaaf RJ, Adriaenssens T, et al. Randomized multi-center trial investigating the angiographic outcome of hybrid sirolimus-eluting stents with biodegradable polymer against everolimus-eluting stents with durable polymer in chronic total occlusions (PRISON IV). JACC Cardiovasc Interv. 2017;10(2):133-43.

30. Pendyala LK, Yin X, Li J, Chen JP, Chronos N, Hou D. The first-generation drug-eluting stents and coronary endothelial dysfunction. JACC Cardiovasc Interv. 2009;2(12):1169-77.

31. Serruys PW, Chevalier B, Sotomi Y, et al. Comparison of an everolimus-eluting bioresorbable scaffold with an everolimus-eluting metallic stent for the treatment of coronary artery stenosis (ABSORB II): a 3 year, randomised, controlled, single-blind, multicentre clinical trial. Lancet. 2016;388(10059):2479-91.

32. Ali ZA, Maehara A, Généreux $P$, et al. Optical coherence tomography compared with intravascular ultrasound and with angiography to guide coronary stent implantation (ILUMIEN III: OPTIMIZE PCI): a randomised controlled trial. Lancet. 2016;388(10060):2618-28.

33. Bech GJ, De Bruyne B, Pijls NH, et al. Fractional flow reserve to determine the appropriateness of angioplasty in moderate coronary stenosis a randomized trial (DEFER). Circulation. 2001;103(24):2928-34.

34. Tonino PAL, De Bruyne B, Pijls NHJ, et al. Fractional flow reserve versus angiography for guiding percutaneous coronary intervention (FAME). N Eng J Med. 2009;360:213-24.

35. De Bruyne B, Pijls NHJ, Kalesan B, et al. Fractional flow reserve-guided PCI versus medical therapy in stable coronary disease (FAME 2). N Engl J Med. 2012;367:991-1001.

36. Rioufol G, Mewton N, Rabilloud M, et al. FUTURE trial ((Does FFR guided treatment strategy in patients with multivessel CAD improve prognosis 
compared to traditional treatment). Presented at American Heart Association Scientific Session 2016. November 14, 2016. New Orleans, LA.

37. Brinton T. DISRUPT CAD: a multicenter, prospective, single-arm study of percutaneous lithoplasty prior to stent implantation in heavily calcified coronary lesions. Presented at Transcatheter Cardiovascular Therapeutics, 2016. Washington DC, October 31, 2016.

38. Leon MB, Smith CR, Mack M, et al. Transcatheter aortic-valve implantation for aortic stenosis in patients who cannot undergo surgery. PARTNER Trial Investigators. $\mathrm{N}$ Eng $\mathrm{J}$ Med. 2010;363(17):1597-607.

39. Leon MB, Smith CR, Mack MJ, et al. Transcatheter of surgical aortic-valve replacement in intermediate-risk patients. PARTNER 2 Investigators. $\mathrm{N}$ Eng J Med. 2016;374(17):1609-20.

40. Werner N. Patients at intermediate surgical risk undergoing isolated interventional or surgical aortic valve replacement for severe symptomatic aortic valve stenosis. One year results from the German Aortic Valve Registry (GARY). Presented at American Heart Association Scientific Sessions 2016. November 13, 2016.

41. Hansson NC, Grove EL, Andersen HR, et al. Transcatheter aortic valve thrombosis: incidence, predisposing factors, and clinical implications. J Am Coll Cardiol. 2016;68(19):2059-69.

42. Kapadia SR, Kodali S, Makkar R, et al. Protection against cerebral embolism during transcatheter aortic valve replacement. J Am Coll Cardiol. 2017;69(4):367-77.

43. Reddy V, Price M, Valderrabano M, et al. Long-term event rates for left atrial appendage closure with WATCHMAN: PROTECT AF 5 Year and PREVAIL 3 year follow-up. Presented at Transcatheter Cardiovascular Therapeutics, 2016. Washington, DC.

44. Thaler DE. RESPECT: final long-term outcomes from a prospective, randomized trial of PFO closure in patients with cryptogenic stroke. Presented at Transcatheter Cardiovascular Therapeutics, 2016. Washington, DC.

45. Schauer P. Bariatric surgery versus intensive medical therapy for type 2 diabetes mellitus. STAMPEDE (Surgical therapy and medications potentially eradicate diabetes efficiently). Presented at American College of Cardiology Conference 2016. Presented at American Heart Association Scientfic Sessions, November 2016.

46. Bentley-Lewis R, Aguilar D, et al. Rational, design and baseline characteristics in evaluation of
LIXisenatide in acute coronary syndrome, a longterm cardiovascular endpoint trial of lixisenatide versus placebo. Am Heart J. 2015;169(5):631-8.

47. Marso AP, Daniels G, et al. Liraglutide and cardiovascular outcomes in type 2 diabetes. $\mathrm{N}$ Eng J Med. 2016;375:311-22.

48. Marso SP, Bain SC, Consoli A, for the SUSTAIN-6 Investigators, et al. Semaglutide and cardiovascular outcomes in patients with type 2 diabetes. $\mathrm{N}$ Engl J Med. 2016;375:1834-44.

49. Nicholls SJ, Puri R, Anderson T, et al. Effect of evolocumab on progression of coronary disease in statin-treated patients: the GLAGOV randomized clinical trial. JAMA. 2016;316(22):2373-84.

50. Moriarty P, Parhofer K, Babirak SP, et al. Alirocumab in patients with heterozygous familial hypercholesterolaemia undergoing lipoprotein apheresis: the ODYSSEY ESCAPE trial. Eur Heart J. 2016;37(48):3588-95.

51. Nissen SE, Stroes E, et al. Efficacy and tolerability of evolocumab vs ezetimibe in patients with muscle-related statin intolerance: the GAUSS-3 randomized clinical trial. JAMA. 2016;315(15):1580-90.

52. Ray KK, Landmesser U, Leiter LA, et al. Inhibition of PCSK9 synthesis via RNA interference: 90 day data from Orion-1: a multi-centre phase- 2 randomized controlled trial. Presented at American Heart Association Scientfic Sessions. November 15, 2016. New Orleans, LA.

53. Nicholls S. A study of Evacetrapid in high-risk vascular disease. Presented at American College of Cardiology Conference, 2016.

54. Bosch J, Lonn E, Yusuf S; HOPE-3 Investigators. The effect of blood pressure and cholesterol lowering on cognition. Program and abstracts of the American Heart Association 2016 Scientific Sessions; November 13, 2016; New Orleans, Louisiana. (Abstract LBCT-01).

55. Domburg RV. Optimal cardiac rehabilitation following acute coronary syndromes-OPTICARE trial. Presented at ESC Congress, 2016, Rome, Italy.

56. Menown IBA, Shand JA. Recent advances in clinical cardiology. Future Cardiol. 2010;6(1):11-7.

57. Vaduganathan M, Bhatt DL, et al. Proton-pump inhibitors reduce gastrointestinal events regardless of aspirin dose in patients requiring dual antiplatelet therapy. J Am Coll Cardiol. 2016;67(14):1661-71.

58. Guillaume C, Cuisset T, et al. Platelet function monitoring to adjust antiplatelet therapy in elderly 
patients stented for an acute coronary syndrome (ANTARTIC): an open label, blinded endpoint, randomised controlled superiority trial. Lancet. 2016;388:2015-21.

59. Motovska A, Hlinomaz O, et al. Prasugrel versus ticagrelor in patients with acute myocardial infarction treated with primary percutaneous coronary intervention multicenter randomized PRAGUE-18 study. Circulation. 2016;134:1603-12.

60. Hiatt WR, Fowkes FG, et al. Ticagrelor versus clopidogrel in symptomatic peripheral artery disease. N Eng J Med. 2017;376(1):32-40.

61. Desai AS, Vardeny O, et al. Reduced risk of hyperkalemia during treatment of heart failure with mineralocorticoid receptor antagonists by use of sacubitril/valsartan compared with enalapril; a secondary analysis of the PARADIGM-HF trial. JAMA Cardiol. 2017;2(1):79-85.

62. McMurray JJV, Krum H, et al. Aliskiren, enalapril, or aliskiren and enalapril in heart failure. N Eng J Med. 2016;374:1521-32.

63. Butler J, Bavry A. Aldosterone targeted neurohormonal combined with natriuresis therapy -heart failure-ATHENA-HF. Presented at American Heart Association Scientfic Sessions, November 2016.

64. Gold MR, Van Veldhusein DJ, et al. Vagus nerve stimulation for the treatment of heart failure. J Am Coll Cardiol. 2016;68(2):149-58.

65. Abraham WT, Adamson PB, Bourge RC, Aaron MF, Costanzo MR, Stevenson LW, et al. Wireless pulmonary artery haemodynamic monitoring in chronic heart failure: a randomized controlled trial. Lancet. 2011;377:658-66.

66. Kleiner Shochat M, Shotan A, et al. Non-invasive lung impedance-guided pre-emptive treatment in chronic heart failure patients: a randomized controlled trail (IMPEDANCE-HF Trial). J Cardiac Fail. 2016;22(9):713-22.

67. Cowie MR. REM-HF: Remote monitoring: an evaluation of implantable devices for management of heart failure patients. European Society of Cardiology 2016 Congress, August 28, 2016, Rome, Italy. (Abstract 1223).

68. Kober L, Thune J, et al. Defibrillator implantation in patients with nonischaemic systolic heart failure. N Eng J Med. 2016;376(13):1221-30.

69. Bardy GH, Lee KL, Mark DB, et al. Amiodarone or an implantable cardioverter-defibrillator for congestive heart failure. $\mathrm{N}$ Eng J Med. 2005;352:225-37.
70. Kadish A, Dyer A, et al. Prophylactic defibrillator implantation in patients with nonischaemic dilated cardiomyopathy. N Eng J Med. 2004;350(21):2151-8.

71. Bartunek J. CHART 1 (Congestive Heart Failure Cardiopoietic regenerative therapy). Presented at ESC congress 2016.

72. Ponikowski P, Van Veldhuisen DJ, et al. Beneficial effects of long term intravenous iron therapy with ferric carboxymaltose in patients with symptomatic heart failure and iron deficiency. Eur Heart J. 2015;36(11):657-68.

73. Veldhuisen DV Ponikowski P, et al. Effect of ferric carboxymaltose on exercise capacity in patients with iron deficiency and chronic heart failure (EFFECT-HF): a randomized, controlled study. Presented at American Heart Association Scientfic Sessions, November 2016.

74. Lewis GD et al. Oral iron repletion effects on oxygen uptake in heart failure (IRONOUT). Presented at American Heart Association Scientific Sessions, 2016.

75. Packer M. Short- and long-term effect of immediate vasodilator therapy in acutely decompensated heart failure: results of the TRUE-AHF trial. Presented at American Heart Association Scientfic Sessions, November 2016.

76. Velazquez EJ, Lee KL, Jones RH, STICHES Investigators, et al. Coronary-artery bypass surgery in patients with ischemic cardiomyopathy. N Eng J Med. 2016;374(16):1511-20.

77. Healey JS, Alings M, et al. Prevalence of sub-clinical atrial fibrillation using an implantable cardiac monitor in patients with cardiovascular risk factors: ASSERT II. Presented at American Heart Association Scientific Sessions, November 2016.

78. Connolly SJ, Milling TJ. Andexanet alfa for acute major bleeding associated with factor Xa inhibitors. N Eng J Med. 2016;375(12):1131-41.

79. 2015 ESC guidelines for the management of acute coronary syndromes in patients presenting without persistent ST-segment elevation. Eur Heart J. 2016;37(3):267-315.

80. Gibson M, et al. An open-label, randomized, controlled, multicenter study exploring two treatment strategies of rivaroxaban and a dose-adjusted oral vitamin $\mathrm{K}$ antagonist treatment strategy in subjects with atrial fibrillation who undergo percutaneous coronary intervention-PIONEER AF-PC. Presented at American Heart Association Scientfic Sessions, November 2016.

81. Nielsen PB, et al. Outcomes associated with oral anticoagulant treatment post-haemorrhagic stroke 
or traumatic intracranial bleeding: a nationwide cohort study on atrial fibrillation patients. Presented at European Society of Cardiology 2016 Congress. August 30, 2016, Rome, Italy. (Abstract 5730).

82. Goette A, Merino JL. Edoxaban versus enoxaparin-warfarin in patients undergoing cardioversion of atrial fibrillation (ENSURE-AF): a randomized, open-label, phase $3 \mathrm{~b}$ trial. Lancet. 2016;388:1995-2003.

83. Kuck K-H, Brugada J. Cryoballoon or radiofrequency ablation for paroxysmal atrial fibrillation. N Eng J Med. 2016;372(23):2235-45. 